\title{
Chemosensitivity is controlled by p63 modification with ubiquitin-like protein ISG15
}

\author{
Young Joo Jeon, Mi Gyeong Jo, Hee Min Yoo, Se-Hoon Hong, Jung-Mi Park, \\ Seung Hyeun Ka, Kyu Hee Oh, Jae Hong Seol, Yong Keun Jung, and Chin Ha Chung
}

School of Biological Sciences, College of Natural Sciences, Seoul National University, Seoul, Republic of Korea.

\begin{abstract}
Identification of the cellular mechanisms that mediate cancer cell chemosensitivity is important for developing new cancer treatment strategies. Several chemotherapeutic drugs increase levels of the posttranslational modifier ISG15, which suggests that ISGylation could suppress oncogenesis. However, how ISGylation of specific target proteins controls tumorigenesis is unknown. Here, we identified proteins that are ISGylated in response to chemotherapy. Treatment of a human mammary epithelial cell line with doxorubicin resulted in ISGylation of the $\mathrm{p} 53$ family protein $\mathrm{p} 63$. An alternative splice variant of $\mathrm{p} 63, \Delta \mathrm{Np} 63 \alpha$, suppressed the transactivity of other p53 family members, and its expression was abnormally elevated in various human epithelial tumors, suggestive of an oncogenic role for this variant. We showed that ISGylation played an essential role in the downregulation of $\Delta \mathrm{Np} 63 \alpha$. Anticancer drugs, including doxorubicin, induced $\Delta \mathrm{Np} 63 \alpha$ ISGylation and caspase-2 activation, leading to cleavage of ISGylated $\Delta \mathrm{Np} 63 \alpha$ in the nucleus and subsequent release of its inhibitory domain to the cytoplasm. ISGylation ablated the ability of $\Delta \mathrm{Np} 63 \alpha$ to promote anchorageindependent cell growth and tumor formation in vivo as well to suppress the transactivities of proapoptotic p53 family members. These findings establish ISG15 as a tumor suppressor via its conjugation to $\Delta \mathrm{Np} 63 \alpha$ and provide a molecular rationale for therapeutic use of doxorubicin against $\Delta \mathrm{Np} 63 \alpha$-mediated cancers.
\end{abstract}

\section{Introduction}

ISG15, the product of IFN-stimulated gene 15, is the first reported ubiquitin-like protein (1). ISG15 is robustly induced by type I IFNs, lipopolysaccharide, and viruses (2-7). Like ubiquitin conjugation, ISGylation of target proteins involves 3-step cascade enzymes: ISG15-activating E1 enzyme (UBE1L), ISG15-conjugating E2 enzyme (UbcH8), and ISG15 E3 ligases (e.g., HERC5 and EFP) (5, 8-11). Proteomics studies have identified more than 300 cellular proteins that are targeted for ISGylation $(10,12,13)$. However, only a dozen of the candidates have been validated as authentic substrates $(14,15)$, and their functional significance remains largely unknown. Intriguingly, UBE1L has been suggested to serve as a tumor suppressor, since it is not detectable in several human lung cancer cell lines, colorectal adenocarcinoma cell line SW480, and leukemia cell lines (16-18). Furthermore, its overexpression inhibits the growth of human bronchial epithelial cells and lung cancer cells (19). However, it remains unknown how ISGylation of specific target proteins affects cellular processes involved in the control of tumorigenesis.

The p53 protein family includes 3 transcription factors, p53, p63, and p73, all of which can induce cell cycle arrest and apoptosis, thus functioning as tumor suppressors. These proteins can be generated as different isotypes, as their genes have 2 different promoters (20). For example, use of the dual promoters in the $p 63$ gene generates 2 types of transcripts: one encodes the $\mathrm{p} 63$ proteins that have an $\mathrm{N}$-terminal transactivation (TA) domain, whereas the other encodes the proteins lacking TA domain $(\Delta \mathrm{N})$. In addition, both TA and $\Delta \mathrm{N}$ transcripts are differentially spliced at their $3^{\prime}$ ends to generate the $\mathrm{p} 63$ proteins with unique $C$ termini designated $\alpha, \beta, \gamma, \delta$, and $\varepsilon(21)$. Similar to p53, TAp63 isotypes activate transcription from p53-responsive

Conflict of interest: The authors have declared that no conflict of interest exists. Citation for this article: J Clin Invest. 2012;122(7):2622-2636. doi:10.1172/JCI61762. genes, which induce cell cycle arrest and apoptosis, thus also functioning as tumor suppressors $(22,23)$. In addition, mice heterozygous for mutations in both $p 53$ and $p 63$ were shown to display higher tumor burden and metastasis compared with $p 53^{+/-}$mice (24). Conversely, it was also shown that $p 63^{+/-}$mice are not tumor prone, and mice heterozygous for both $p 63$ and $p 53$ have fewer tumors than do $p 53^{+/-}$mice (25). Importantly, TAp63 isotypes were shown to act as mediators of cell senescence independently of p53 (26). Thus, it appears that TAp63 can inhibit tumorigenesis not only by promoting expression of p53-responsive genes, but also by inducing senescence.

The $\mathrm{p} 63 \alpha$ isotypes contain a transactivation-inhibitory (TI) domain in their $\mathrm{C}$ termini, which can interact with TA domain and suppress TAp63-mediated transactivation (27). Therefore, the smaller TAp $63 \beta$ and TAp63 $\gamma$, which lack TI domain, are more transcriptionally active than TAp $63 \alpha$. Recently, the mechanism by which TI domain inhibits the activity of TAp $63 \alpha$ has been demonstrated (28). In unstressed oocytes, TAp63 $\alpha$ is kept in a dimeric, closed, and inactive conformation. Both the N-terminal TA and C-terminal TI domains are required to form this closed state. Upon activation, such as by $\gamma$-irradiation, a conformational switch is triggered to release the inhibitory interactions, allowing TAp63 $\alpha$ to tetramerize and interact with the transcriptional machinery through its TA domain.

Having TI domain but lacking TA domain, $\Delta \mathrm{Np} 63 \alpha$ is hence capable of suppressing transactivation by TA isotypes $(27,29,30)$. Accordingly, $\Delta \mathrm{Np} 63 \alpha$ inhibits the activity of p53 family members in a dominant-negative manner, contributing to cell proliferation and tumorigenesis. $\Delta \mathrm{Np} 63 \alpha$ is the most abundant p63 isotype in many proliferating epithelial tissues (31-33). Importantly, its expression is frequently amplified in human epithelial cancers, such as squamous cell carcinomas, advanced cervical carcinomas, urothelial bladder carcinomas, and human breast carcinomas, supporting its role in tumorigenesis (34-37). Moreover, dysregulated expression of p63 frequently occurs in conjunction with ampli- 
fication of its genomic region at 3q27-29 in a subset of human epithelial cancers $(36,38)$. In addition to the role of $\Delta \mathrm{Np} 63 \alpha$ as a negative regulator of TA isotypes, $\Delta \mathrm{Np} 63$ can activate subsets of target genes, likely due to the presence of a second TA domain (39-43). For example, $\Delta \mathrm{Np} 63 \alpha$ initially induces expression of the extracellular matrix component Fras1 for maintaining integrity of the epidermal-dermal interface at the basement membrane and subsequently IкB kinase- $\alpha$ for initiation of epidermal terminal differentiation (44). It was also shown that knockdown of p63 causes downregulation of cell adhesion-associated genes in mammary epithelial cells and keratinocytes, whereas overexpression of $\Delta \mathrm{Np} 63 \alpha$ isoforms upregulates cell adhesion molecules (31). In addition, $\Delta \mathrm{Np} 63 \alpha$ was shown to target the chromatin remodeling Lsh protein to drive skin stem cell proliferation and tumorigenesis (45). Thus, $\Delta \mathrm{Np} 63 \alpha$ also plays a key role in maintaining epidermal morphogenesis and homeostasis.

Several studies have shown that modification of $\Delta \mathrm{Np} 63 \alpha$ by ubiquitin and SUMO is involved in its destabilization (46-49). Itch and NEDD 4 act as E3 ligases for $\triangle \mathrm{Np} 63 \alpha$ ubiquitination (50-52). WWP1 also serves as an ubiquitin E3 ligase for both $\Delta \mathrm{Np} 63 \alpha$ and TAp63 $\alpha$ (53). However, it was recently shown that WWP1 binds specifically to $\Delta \mathrm{Np} 63 \alpha$, but does not trigger proteasome-dependent degradation (54). WWP1-dependent ubiquitination occurs through formation of K63-linked polyubiquitin chains, leading to the control of $\Delta \mathrm{Np} 63 \alpha$-dependent transcription.

Chemosensitivity is a key to the action of anticancer drugs in clinical applications. Therefore, it is important to dissect the biochemical processes of relevant modifications in response to anticancer drugs and understand the functional implications of these regulatory processes. A good strategy for addressing the function of ISGylation in the control of tumorigenesis is to search target proteins for ISGylation in the context of treatment with chemotherapeutic agents. Here, we identified $\Delta \mathrm{Np} 63 \alpha$ as a target for ISGylation in response to anticancer drugs such as doxorubicin and camptothecin, a finding we believe to be novel. Furthermore, caspase- 2 was found to specifically cleave ISGylated $\Delta \mathrm{Np} 63 \alpha$, resulting in release of its C-terminal TI domain to the cytoplasm. This export abrogates the dominant-negative function of $\Delta \mathrm{Np} 63 \alpha$ toward transcriptionally active TA isotypes, leading to tumor suppression. TAp63 $\alpha$ was also ISGylated and cleaved by caspase-2, and its TI domain was released to the cytoplasm, thus yielding a transcriptionally active form of TAp $63 \alpha$. These findings demonstrated that ISGylation of p63 isotypes plays a pivotal role in the control of tumorigenesis in response to chemotherapeutic agents.

\section{Results}

Chemotherapeutic drugs induce ISGylation of $\Delta N p 63 \alpha$. Cancer chemotherapeutics have been shown to increase the levels of ISG15 and its conjugates, suggesting a role for ISG15 as a tumor suppressor. For example, treatment with camptothecin leads to an increase in the level of ISG15 mRNA (55). Furthermore, treatment with both IFNs and camptothecin causes synergistic killing of colorectal cancer xenografts in nude mice (56). In order to clarify the role of ISG15 in tumor suppression, it is essential to identify target proteins that are ISGylated in response to chemotherapeutic drugs. Therefore, we first examined whether the drugs can induce the components of ISGylation machinery in a nonmalignant but highly proliferative mammary epithelial cell line, MCF10A. Both doxorubicin and camptothecin, but not cisplatin, apparently ele- vated the mRNA levels of ISG15, UBE1L, and UbcH8 (Figure 1A). This increase was observable from about 6 hours and became maximal by about 24 hours after drug treatment. Consistently, doxorubicin and camptothecin, but not cisplatin, dramatically elevated their protein levels, leading to induction of cellular protein ISGylation (Figure 1B).

Since doxorubicin increased the levels of ISGylated cellular proteins more evidently than did camptothecin, we used doxorubicin to identify target proteins for ISGylation. MCF10A cells cultured with and without doxorubicin were subjected to immunoaffinity purification using anti-ISG15 antibody-immobilized resins. Proteins bound to the resins were subjected to SDS-PAGE (Figure 1C) and mass spectrometry. Since the identified proteins included p 63 (Supplemental Table 1; supplemental material available online with this article; doi:10.1172/JCI61762DS1), and since $\Delta \mathrm{Np} 63 \alpha$ is an oncoprotein (36), we examined whether $\Delta \mathrm{Np} 63 \alpha$ could be ISGylated. Overexpression of $\Delta \mathrm{Np} 63 \alpha$ with UBE1L, UbcH8, and ISG15 (referred to as ISG15-conjugating system) led to the appearance of at least 2 ISGylated $\Delta \mathrm{Np} 63 \alpha$ bands (Figure 1D). IP analysis also revealed that doxorubicin and camptothecin, but not cisplatin, markedly induced ISGylation of endogenous $\Delta \mathrm{Np} 63 \alpha$ in MCF10A cells (Figure 1E). Furthermore, as shown in Figure 1F, doxorubicin and camptothecin, but not cisplatin, robustly induced ISGylation of endogenous $\Delta \mathrm{Np} 63 \alpha$ in cancer cells, including HNSCC013 (head and neck squamous carcinoma), HCC1937 (human breast carcinoma), and FaDu (pharyngeal squamous cell carcinoma), all of which are known to highly express $\Delta \mathrm{Np} 63 \alpha(36,37)$. In addition, both doxorubicin and camptothecin elevated the mRNA levels of ISG15, UBE1L, and UbcH8 in the cancer cells (Supplemental Figure 1 and data not shown). To determine whether the inability of cisplatin to induce $\Delta \mathrm{Np} 63 \alpha$ ISGylation is due to instability of the drug itself, the cancer cells were treated with increasing amounts of cisplatin. No sign of $\Delta \mathrm{Np} 63 \alpha$ ISGylation was detected at all concentrations tested (Supplemental Figure 2). These results indicate that $\Delta \mathrm{Np} 63 \alpha$ is a target protein for ISGylation in cancer cells as well as in rapidly proliferating normal epithelial cells in response to doxorubicin or camptothecin, but not cisplatin.

Identification of ISGylation sites in $\Delta N p 63 \alpha$. To determine ISGylation sites, various deletions of $\Delta \mathrm{Np} 63 \alpha$ were expressed in HeLa cells with ISG15-conjugating system (Figure 2A). All F3-containing constructs (i.e., having the aa sequence 1-210) and F5 (aa 211-586), but not F4 (aa 331-586), were conjugated by ISG15, which indicates that F3 and the sequence 211-330 have ISGylation sites. Among the 8 lysine residues in F3, replacement of residue 139 with arginine, but not other K-to-R substitutions, prevented ISGylation (Figure 2B). Likewise, among the 8 residues in the aa sequence 211-330 in F5, K324R, but not the other substitutions, blocked ISGylation (Figure $2 \mathrm{C}$ ). These results indicate that $\Delta \mathrm{Np} 63 \alpha$ has 2 ISGylation sites. To confirm this finding, we replaced both of the lysine residues by arginine in full-length $\Delta \mathrm{Np} 63 \alpha$. The double K-to-R mutation (referred to herein as KR) completely blocked $\Delta \mathrm{Np} 63 \alpha$ ISGylation (Figure $2 \mathrm{D}$ ), which indicates that K139 and K324 are the ISGylation sites in $\Delta \mathrm{Np} 63 \alpha$.

ISGylation is required for caspase-mediated cleavage of $\Delta N p 63 \alpha$. Notably, the level of $\Delta \mathrm{Np} 63 \alpha$ in cell lysates decreased in parallel with an increase in ISGylated $\Delta \mathrm{Np} 63 \alpha$ (Figure 1, E and F). To determine whether ISGylation of $\Delta \mathrm{Np} 63 \alpha$ affects its stability, $\Delta \mathrm{Np} 63 \alpha$ and KR were complemented to H1299 cells, a non-small-cell lung carcinoma cell line that does not express any detectable p63 or 

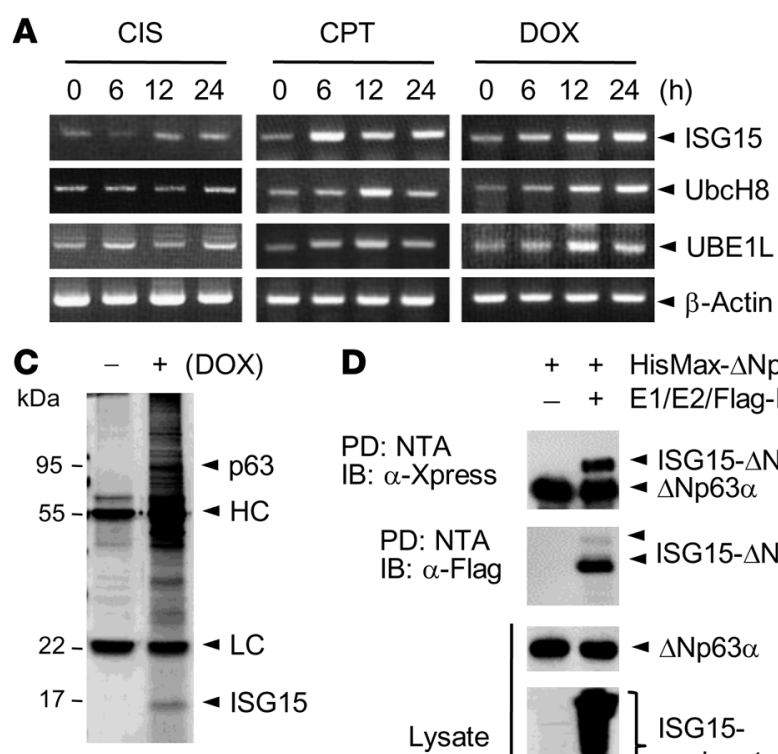

D

PD: NTA

IB: $\alpha$-Xpress

PD: NTA

IB: $\alpha$-Flag

+ + HisMax- $\Delta$ Np63 $\alpha$

- + E1/E2/Flag-ISG15

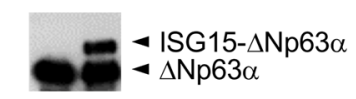

B
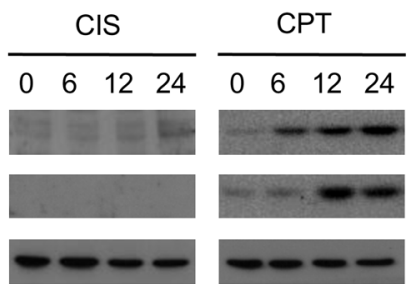

\begin{tabular}{llll}
\multicolumn{4}{c}{ DOX } \\
\hline 0 & 6 & 12 & 24
\end{tabular}
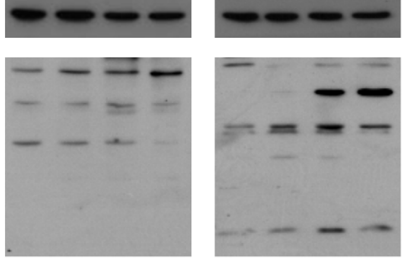

Lysate
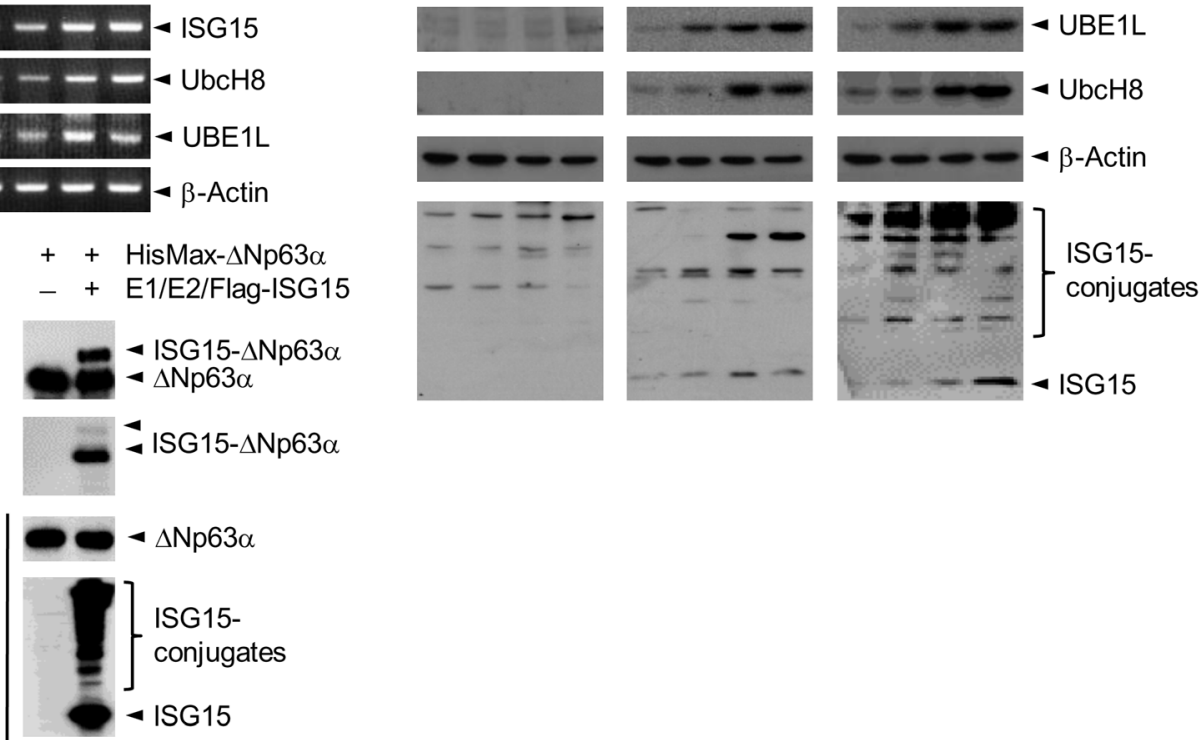

E

CIS

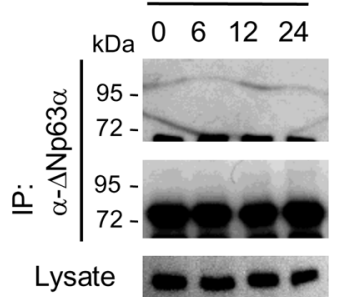

\begin{tabular}{llll}
\multicolumn{3}{c}{ CPT } \\
\hline 0 & 6 & $12 \quad 24$
\end{tabular}

DOX

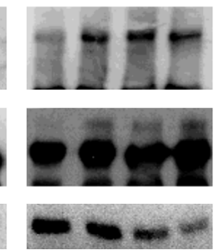

$\begin{array}{llll}0 & 6 & 12 & 24\end{array}$

(h)

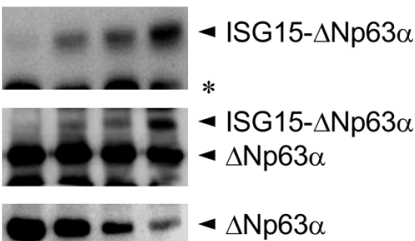

$\mathbf{F}$

HNSCC013

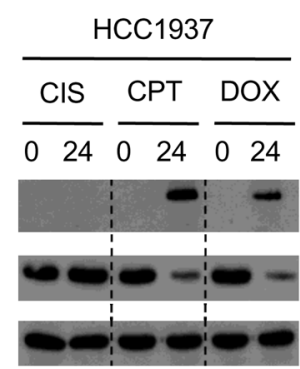

$\frac{\text { FaDu }}{\frac{\mathrm{CIS}}{0 \quad 24} \frac{\mathrm{CPT}}{024} \frac{\mathrm{DOX}}{024}(\mathrm{~h})}$

$\begin{array}{ll}\text { IP: } \alpha-\Delta N p 63 \alpha & 95- \\ \text { IB: } \alpha-I S G 15 & 72-\end{array}$

$\frac{\text { CIS }}{24} \frac{\text { CPT }}{024} \frac{\text { DOX }}{024}$
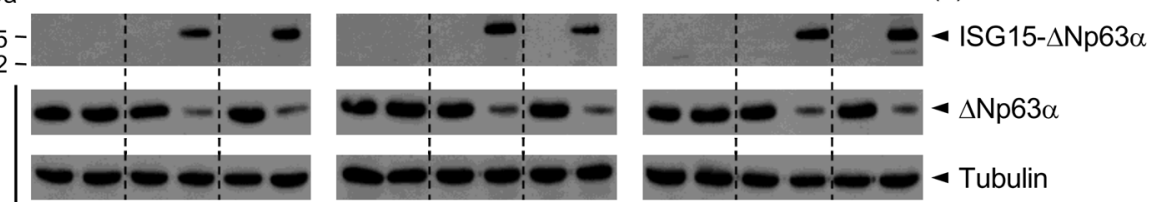

Figure 1

Induction of ISG15-conjugating systems and ISGylation of $\triangle N p 63 \alpha$ in response to chemotherapeutic drugs. (A) MCF10A cells were treated with $20 \mu \mathrm{M}$ cisplatin (CIS), $0.25 \mu \mathrm{M}$ camptothecin (CPT), or $0.1 \mu \mathrm{M}$ doxorubicin (DOX). Total RNAs were prepared and subjected to RT-PCR using the probes for ISG15, UbcH8, UBE1L, and $\beta$-actin. (B) Cell lysates from A were subjected to IB analysis. (C) Lysates from cells cultured without or with doxorubicin for 12 hours were incubated with anti-ISG15 antibody-immobilized protein A-conjugated Sepharose beads. Bound proteins were eluted and subjected to SDS-PAGE followed by silver staining. HC and LC, heavy and light chains of IgG, respectively. (D) HisMax-tagged $\triangle \mathrm{Np63} \alpha$ was expressed with or without UBE1L (E1), UbcH8 (E2), and Flag-tagged ISG15 in HeLa cells. Cell lysates were subjected to pulldown (PD) with NTA resins followed by IB with anti-Xpress or anti-Flag antibody. They were also directly probed with the same antibodies. (E) MCF10A cells incubated with anticancer drugs were subjected to IP with anti- $\Delta$ Np63 $\alpha$ antibody followed by IB with anti- $\Delta$ Np $63 \alpha$ or anti-ISG15 antibody. Asterisk indicates IgG heavy chain. (F) HNSCC013, HCC1937, and FaDu cells treated with anticancer drugs were subjected to IP as in E followed by IB with anti-ISG15 antibody.

p53 protein (30). Doxorubicin induced the cleavage of $\Delta \mathrm{Np} 63 \alpha$, but not KR, with generation of the $55-\mathrm{kDa}$ product (Figure $3 \mathrm{~A}$ ). Furthermore, the $\triangle \mathrm{Np} 63 \alpha$ level declined in $U B E 1 L^{+/+}$, but not $U B E 1 L^{-/-}$, mouse embryonic fibroblasts (MEFs) upon doxorubicin treatment (Figure 3B). These results indicate that ISGylation of $\Delta \mathrm{Np} 63 \alpha$ is required for its cleavage.
$\Delta \mathrm{Np} 63 \alpha$ can be degraded by ubiquitin-proteasome system $(46,47,50-52)$ as well as by caspase, although it was unknown which caspases specifically degrade $\Delta \mathrm{Np} 63 \alpha(30,57)$. Therefore, we examined whether either or both proteases are involved in doxorubicin-induced $\Delta \mathrm{Np} 63 \alpha$ cleavage in $\mathrm{H} 1299$ cells. $\Delta \mathrm{Np} 63 \alpha$ cleavage was prevented by Z-VAD-fmk, a pan-caspase inhibitor, 
A

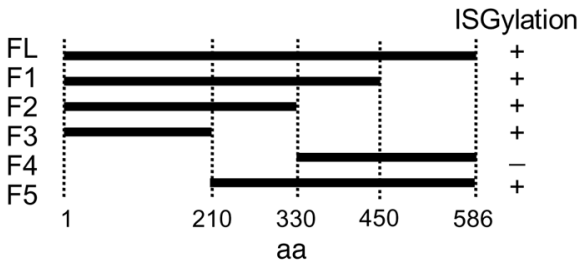

C

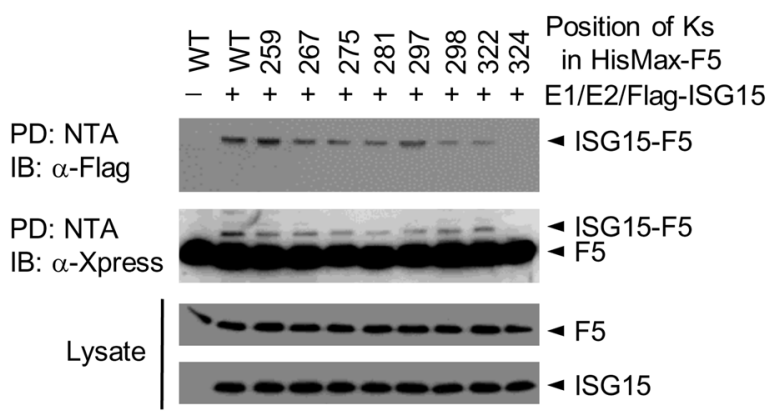

B

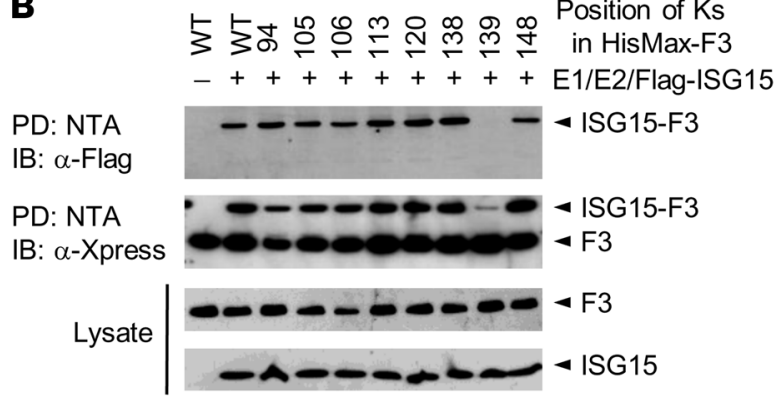

D $\quad$ 与舀 HisMax- $-\mathrm{N} p 63 \alpha$

+++ E1/E2/Flag-ISG15

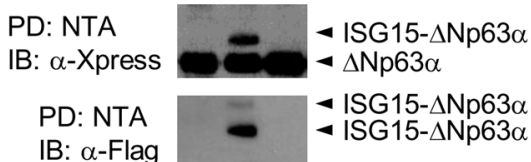

B: $\alpha$-Flag

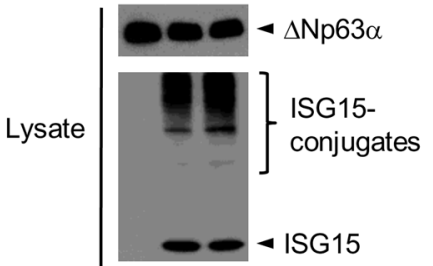

Figure 2

Identification of ISGylation sites in $\Delta \mathrm{Np} 63 \alpha$. (A) Deletions of $\Delta \mathrm{Np} 63 \alpha$ were generated, and their $\mathrm{N}$ termini were tagged with HisMax. Whether each deletion was modified by ISG15 is indicated at right. FL, full-length. (B-D) HisMax-tagged (B) F3, (C) F5, and (D) $\Delta$ Np63 $\alpha$ and KR were expressed in HeLa cells with UBE1L, UbcH8, and Flag-tagged ISG15. Cell lysates were subjected to pulldown with NTA resins followed by IB with anti-Xpress or anti-Flag antibody.

but not by MG132, a proteasome inhibitor (Figure 3C). Moreover, endogenous $\triangle \mathrm{Np} 63 \alpha$ in MCF10A cells was cleaved into $\mathrm{N}$-terminal $55-\mathrm{kDa}$ and C-terminal $20-\mathrm{kDa}$ fragments, and this cleavage was abolished by Z-VAD-fmk (Figure 3D). The inhibitor also prevented camptothecin-induced cleavage of endogenous $\Delta \mathrm{Np} 63 \alpha$ (Supplemental Figure 3A). On the other hand, the level of $\Delta \mathrm{Np} 63 \alpha$ remained unchanged regardless of treatment with cisplatin, Z-VAD-fmk, or both (Supplemental Figure 3B). These results indicate that ISGylated $\Delta \mathrm{Np} 63 \alpha$ is cleaved by caspases, but not by proteasome.

Caspase- 2 is responsible for $\Delta N p 63 \alpha$ cleavage. Caspase- 2 is required for stress-induced apoptosis $(58,59)$. Furthermore, caspase-2-deficient germ cells and oocytes are resistant to cell death after treatment with chemotherapeutic drugs (60). In addition, caspase- 2 is the only caspase that is present constitutively in the nucleus (61-63). Based on these reports, we tested whether caspase- 2 cleaves $\Delta \mathrm{Np} 63 \alpha$. A caspase-2-specific shRNA (shCasp-2), but not a caspase-9-specific shRNA (shCasp-9) or nonspecific shRNA (shNS), strongly inhibited doxorubicin-induced cleavage of $\Delta \mathrm{Np} 63 \alpha$ (Figure $4 \mathrm{~A})$. Furthermore, $\Delta \mathrm{Np} 63 \alpha$ was degraded by overexpression of pro-caspase-2, but not by that of its catalytically inactive mutant, which was generated by substituting the active site C303 with alanine (Figure 4B). These results indicate that caspase- 2 is responsible for $\Delta \mathrm{Np} 63 \alpha$ cleavage.

Notably, overexpressed pro-caspase- 2 in the absence of doxorubicin hydrolyzed $\Delta \mathrm{Np} 63 \alpha$ (Figure $4 \mathrm{~B}$ ). Although pro-caspase-2 is known to be autoactivated when overexpressed $(64,65)$, this result seemed contradictory to our finding that doxorubicin-induced ISGylation was required for caspase-dependent $\Delta \mathrm{Np} 63 \alpha$ cleavage (Figure 3, A and B). One possible explanation could be that doxorubicin induces not only $\Delta \mathrm{Np} 63 \alpha$ ISGylation, but also activation of endogenous caspase-2, and the activated enzyme preferentially interacts with and acts on ISGylated $\Delta \mathrm{Np} 63 \alpha$ over its unmodified form. To test this possibility, H1299 cells complemented with $\Delta \mathrm{Np} 63 \alpha$ or KR were incubated with and without doxorubicin. Indeed, doxorubicin induced endogenous caspase- 2 activation (Figure 4, A and C). Moreover, the activated caspase- 2 interacted with $\Delta \mathrm{Np} 63 \alpha$, but not with its KR mutant (Figure 4C), consistent with our finding that $\mathrm{KR}$ was resistant to proteolysis (Figure 3A). These results indicate that ISGylated $\Delta \mathrm{Np} 63 \alpha$ is preferentially cleaved by activated endogenous caspase- 2 .

To confirm whether doxorubicin induces the activation of endogenous caspase-2, we administered the drug in HCC1937, HNSCC013, and FaDu cancer cells, which increased activated caspase-2 levels in a time-dependent manner (Figure 4D). Moreover, $\Delta \mathrm{Np} 63 \alpha$ cleavage was abolished by incubation with Z-VDVADfmk, a caspase-2-specific inhibitor (Figure 4E). These results indicate that doxorubicin induces caspase- 2 activation and that the activated protease cleaves ISGylated $\Delta \mathrm{Np} 63 \alpha$.

Determination of caspase- 2 cleavage sites in $\Delta N p 63 \alpha$. Since doxorubicin treatment led to generation of an approximately $55-\mathrm{kDa}$ fragment from $\Delta \mathrm{Np} 63 \alpha$ (Figure $3, \mathrm{~A}$ and $\mathrm{D}$ ), each of 6 aspartic acid residues located around the $\mathrm{C}$-terminal region of the fragment was replaced by alanine. Overexpression of pro-caspase- 2 

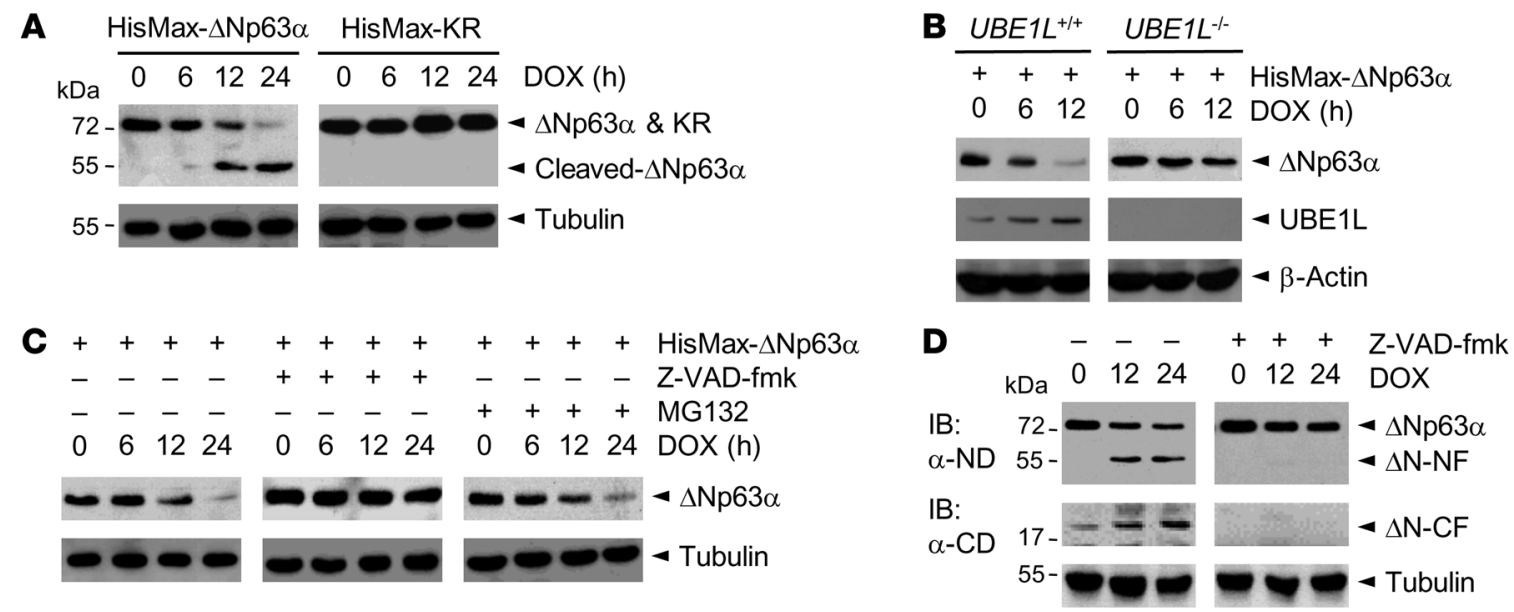

Figure 3

ISGylation of $\Delta \mathrm{Np} 63 \alpha$ is required for its caspase-mediated cleavage. (A) H1299 cells complemented with HisMax-tagged $\Delta$ Np63 $\alpha$ or KR were incubated with doxorubicin, then subjected to IB with anti-Xpress antibody. (B) UBE1 $L^{+/+}$or UBE1 $L^{-/-}$MEFs expressing HisMax-tagged $\triangle N p 63 \alpha$ were incubated with doxorubicin followed by IB with anti-Xpress or anti-UBE1L antibody. (C) H1299 cells complemented with HisMax-tagged $\Delta \mathrm{Np} 63 \alpha$ were incubated as in A, but in the absence or presence of $100 \mu \mathrm{M}$ Z-VAD-fmk or $10 \mu \mathrm{M}$ MG132. (D) MCF10A cells were incubated with doxorubicin in the absence or presence of Z-VAD-fmk followed by IB with anti-ND and anti-CD antibodies. $\Delta \mathrm{N}-\mathrm{NF}$ and $\triangle \mathrm{N}-\mathrm{CF}, \mathrm{N}$ - and C-terminal fragments of $\Delta \mathrm{Np} 63 \alpha$, respectively.

caused extensive degradation of $\Delta \mathrm{Np} 63 \alpha$ that was attenuated in part by D452A, D469A, and D489A, but not by other substitutions (Figure 4F), which suggests that caspase-2 cleaves multiple sites in $\Delta \mathrm{Np} 63 \alpha$. Indeed, triple D-to-A mutation of these residues (referred to herein as 3DA) completely stabilized $\Delta \mathrm{Np} 63 \alpha$ under conditions of both caspase- 2 overexpression and doxorubicin treatment (Figure 4G), despite the finding that 3DA showed little or no effect on doxorubicin-induced ISGylation of $\Delta \mathrm{Np} 63 \alpha$ (Figure $4 \mathrm{H})$. These data indicate that D452, D469, and D489 of $\Delta \mathrm{Np} 63 \alpha$ are the caspase- 2 cleavage sites. Our results also indicated that caspase- 2 can cleave off the C-terminal fragment containing TI domain from $\Delta \mathrm{Np} 63 \alpha$, and likely also from TAp63 $\alpha$ (Figure 4I).

Previously, it was shown that apoptotic stimuli, such as UV and staurosporine treatment, induce TI domain removal from $\mathrm{p} 63 \alpha$ isotypes by activated caspases, such as caspase-3, and its export to the cytoplasm (30). Furthermore, cleavage of $\Delta \mathrm{Np} 63 \alpha$ relieves its inhibitory effect on TAp63 isotypes, and cleavage of TAp63 $\alpha$ results in production of a TAp 63 protein with enhanced transcriptional activity. Therefore, we examined whether caspase- 3 is also involved in doxorubicin-mediated cleavage of $\Delta \mathrm{Np} 63 \alpha$. Doxorubicin treatment led to generation of activated caspase-3 (Supplemental Figure 4A). However, neither treatment with Z-DEVD-fmk, a caspase-3-specific inhibitor, nor knockdown of caspase-3 attenuated doxorubicin-induced cleavage of $\Delta \mathrm{Np} 63 \alpha$ (Supplemental Figure 4, B and C). In addition, the cleavage site of caspase- 3 in $\Delta \mathrm{Np} 63 \alpha, \mathrm{D} 403$, did not overlap with that of caspase-2 (Supplemental Figure 5). These results indicate that caspase- 2 is the major protease that cleaves $\Delta \mathrm{Np} 63 \alpha$ in response to doxorubicin.

Caspase-2-mediated cleavage of $\Delta N p 63 \alpha$ releases its $T I$ domain to the cytoplasm. TI domain is capable of binding directly to TA domain (27). Therefore, TI domain cleaved off from $\Delta \mathrm{Np} 63 \alpha$ would still be capable of inhibiting the transactivities of TA isotypes, provided it remains in the nucleus. One possible way to overcome this inhibition is the export of cleaved TI domain to the cytoplasm. To test this possibility, we determined the subcellular localization of $\Delta \mathrm{Np} 63 \alpha$ and its cleavage products using antibodies directed against the $\mathrm{N}$ - and C-terminal domains of $\Delta \mathrm{Np} 63 \alpha$ (referred to herein as anti-ND and anti-CD antibodies, respectively). Upon staining with anti-ND antibody, both overexpressed $\Delta \mathrm{Np} 63 \alpha$ and $\mathrm{KR}$ were detected exclusively in the nucleus of H1299 cells, regardless of doxorubicin treatment (Figure 5A). Numbers of cells having the $\mathrm{N}$ - or C-terminal fragment of $\Delta \mathrm{Np} 63 \alpha$ in the nucleus only, the cytoplasm only, and both compartments were counted (Supplemental Figure 6A). However, when stained with anti-CD antibody, $\Delta \mathrm{Np} 63 \alpha$ was found to localize in both cytoplasm and nucleus in doxorubicintreated cells, whereas KR remained in the nucleus regardless of drug treatment (Figure 5B and Supplemental Figure 6B). Like $\mathrm{KR}, 3 \mathrm{DA}$ also remained in the nucleus regardless of doxorubicin treatment (Figure 5C and Supplemental Figure 6C). To confirm these findings, cells prepared as above were also subjected to subcellular fractionation. In the absence of doxorubicin, all of $\Delta \mathrm{Np} 63 \alpha, \mathrm{KR}$, and 3DA were detected predominantly in the nuclear fraction (Supplemental Figure 7A). In the setting of doxorubicin treatment, the level of $\Delta \mathrm{Np} 63 \alpha$ in the nuclear fraction was dramatically decreased, concurrent with an increase in the level of the C-terminal fragments in the cytoplasmic fraction, whereas KR and 3DA remained exclusively in the nuclear fraction. These results suggest that TI domain is exported to the cytoplasm upon caspase-2-mediated cleavage of ISGylated $\Delta \mathrm{Np} 63 \alpha$ in the nucleus in response to doxorubicin.

We next examined subcellular localization of endogenous $\Delta \mathrm{Np} 63 \alpha$ under conditions of caspase- 2 inhibition or depletion by staining HNSCC013 cells with anti-CD antibody. In the absence of Z-VDVAD-fmk, $\Delta \mathrm{Np} 63 \alpha$ was detected predominantly in the cytoplasm of more than $60 \%$ of doxorubicin-treated cells (Figure 5D and Supplemental Figure 6D); in its presence, however, $\Delta \mathrm{Np} 63 \alpha$ remained exclusively in the nucleus. IB analysis also revealed that $Z$-VDVAD-fmk treatment abolished generation 


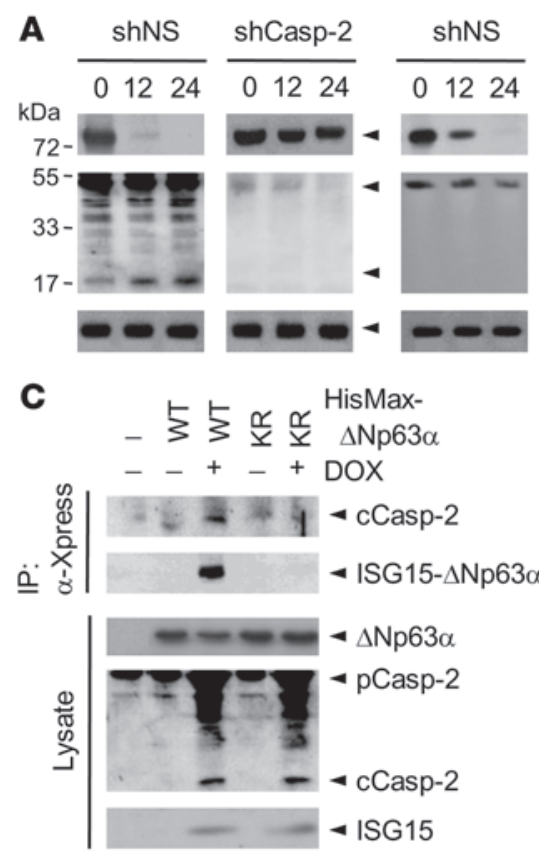

$\mathbf{F}$
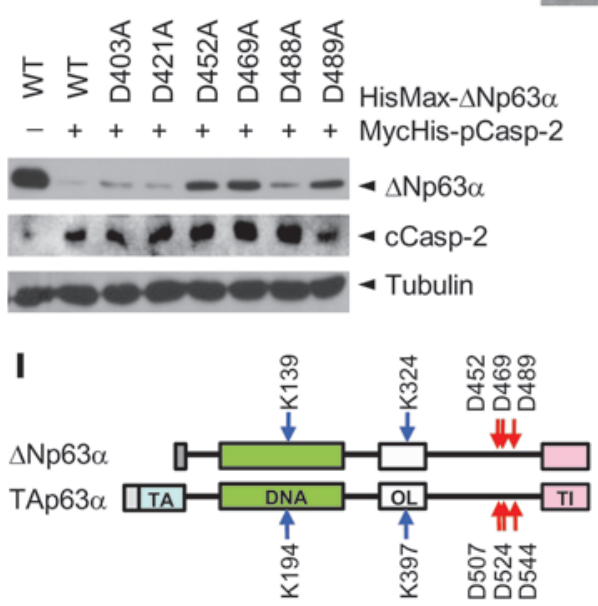

E
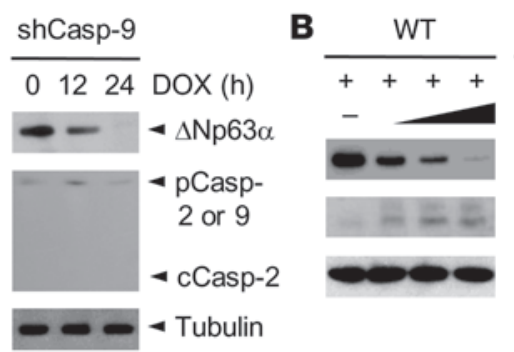
$\frac{\mathrm{Mt}}{++++}$ pCasp-2 ++++ HisMax- $\Delta$ Np63 $\alpha$ MycHis-pCasp-2 $-\Delta \mathrm{Np} 63 \alpha$

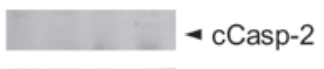

\section{.}
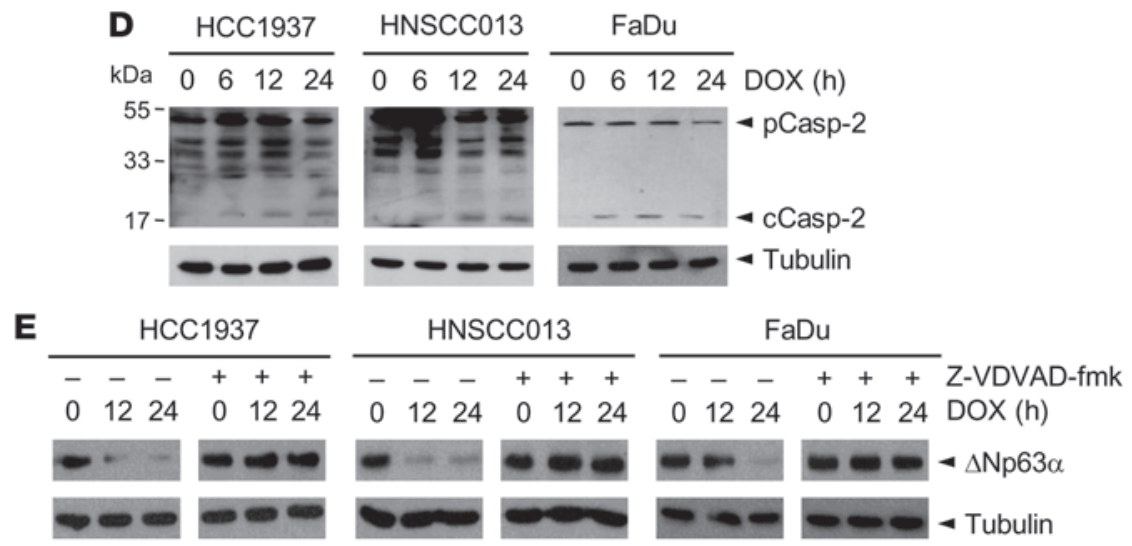

G

H

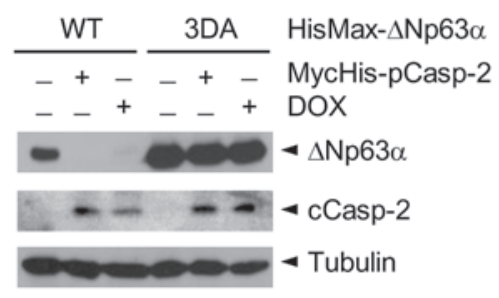

\section{Figure 4}

Cleavage of $\Delta N p 63 \alpha$ by caspase-2, and identification of the cleavage sites. (A) MCF10A cells transfected with shNS, shCasp-2, or shCasp-9 were incubated with doxorubicin followed by IB analysis. pCasp-2 and pCasp-9, pro-caspase-2 and pro-caspase-9, respectively; cCasp-2, cleaved caspase-2. (B) HisMax-tagged $\Delta \mathrm{Np} 63 \alpha$ was expressed in $\mathrm{H} 1299$ cells with MycHis-tagged pro-caspase-2 (WT) or its inactive mutant (Mt). (C) H1299 cells complemented with HisMax-tagged $\Delta$ Np63 $\alpha$ (WT) or KR were incubated with and without doxorubicin. Cell lysates were subjected to IP with anti-Xpress antibody followed by IB with anti-caspase-2 or anti-ISG15 antibody. (D) HCC1937, HNSCC013, or FaDu cells were incubated with doxorubicin followed by IB with anti-caspase-2 antibody. (E) HCC1937, HNSCC013, or FaDu cells were incubated with doxorubicin in the absence or presence of $10 \mu \mathrm{M}$ Z-VDVAD-fmk followed by IB with anti- $\Delta$ Np63 $\alpha$ antibody. (F) HisMax-tagged $\Delta$ Np63 $\alpha$ and its D-to-A mutants were complemented to $\mathrm{H} 1299$ cells with MycHis-tagged pro-caspase-2. Cell lysates were subjected to IB analysis. (G) HisMax-tagged $\Delta$ Np63 $\alpha$ and 3DA were expressed in $\mathrm{H} 1299$ cells with MycHis-tagged pro-caspase-2 in the absence or presence of doxorubicin. (H) H1299 cells complemented with HisMax-tagged 3DA were incubated with and without doxorubicin. Cell lysates were subjected to pulldown with NTA resins followed by IB with anti-Xpress or anti-ISG15 antibody. (I) Domain structures of $\Delta$ Np63 $\alpha$ and TAp63 $\alpha$. Blue arrows denote ISGylation sites; red arrows denote cleavage sites by caspase-2. DNA, DNA-binding; OL, oligomerization.

of the C-terminal fragment by caspase-2 (Figure 5G). Likewise, $\Delta \mathrm{Np} 63 \alpha$ remained exclusively in the nucleus upon knockdown of caspase-2 by shCasp-2 (Figure 5E and Supplemental Figure 6E). IB analysis showed that shCasp-2 efficiently depleted caspase-2 and prevented doxorubicin-induced $\Delta \mathrm{Np} 63 \alpha$ cleavage (Figure $5 \mathrm{H}$ ). ISG15 knockdown by shRNA specific to ISG15 (shISG15) also abolished doxorubicin-mediated cytoplasmic localization of the C-terminal fragment of $\Delta \mathrm{Np} 63 \alpha$ (Figure 5F and Supplemental Figure 6F), and IB analysis showed that shISG15 depleted ISG15 and blocked $\Delta$ Np $63 \alpha$ cleavage (Figure 5I). Subcellular fractionation confirmed that doxorubicin induced export of the C-terminal fragments of $\mathrm{p} 63 \alpha$, such as $\Delta \mathrm{Np} 63 \alpha$ and TAp $63 \alpha$, to 
A
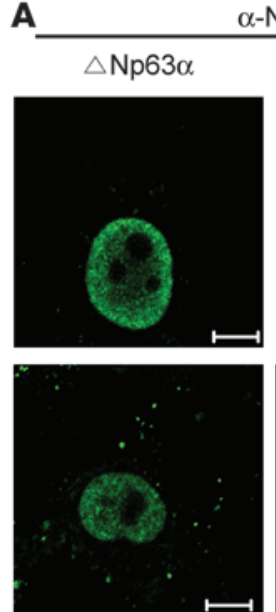

D
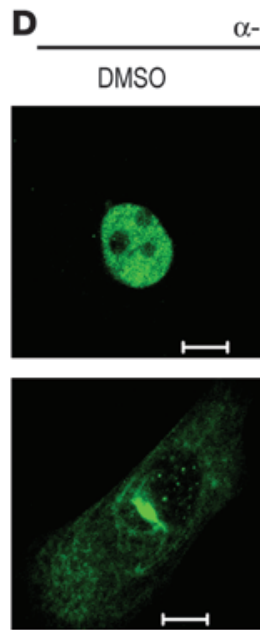

G $-\quad-\quad-++++$ - $f \mathrm{mk}$ $\begin{array}{llllllllll}0 & 6 & 12 & 24 & 0 & 6 & 12 & 24 & \mathrm{DOX} & (\mathrm{h})\end{array}$

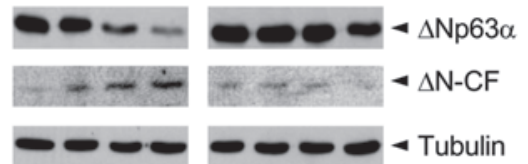

B

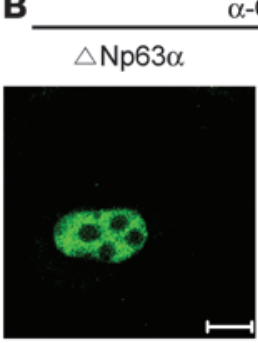

$\alpha-C D$
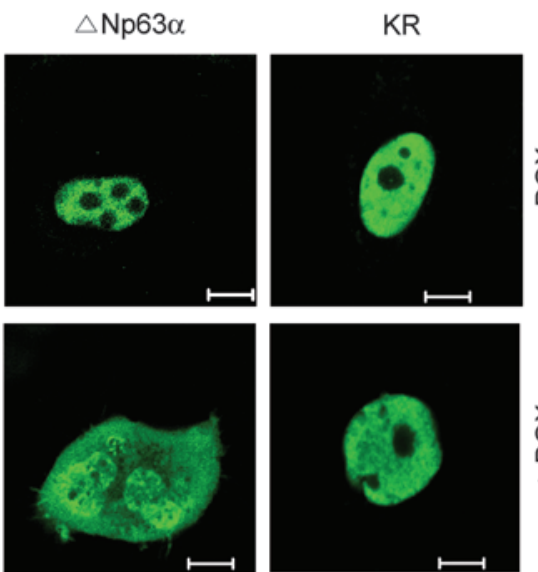

E

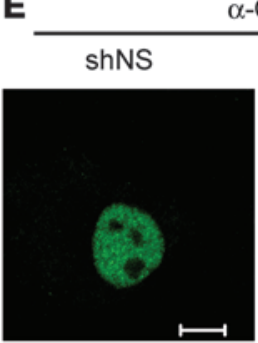

$\alpha-C D$
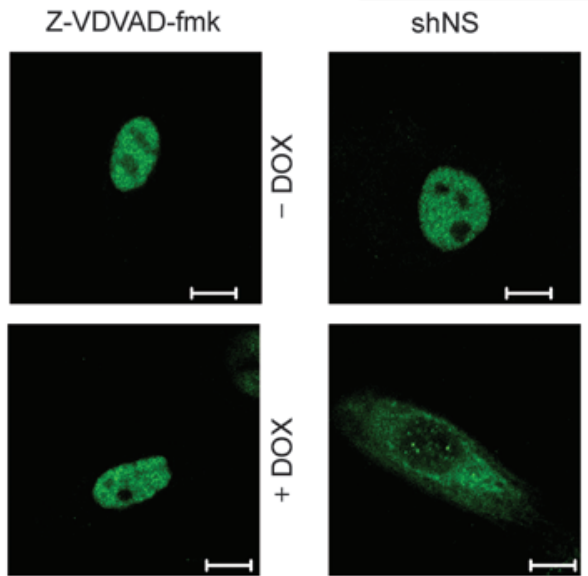

H +++ $\begin{array}{cccc}- & - & - & - \\ 0 & 6 & 12 & 24\end{array}$

$-$

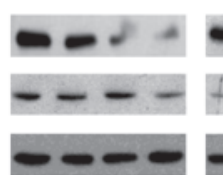

shCasp-2
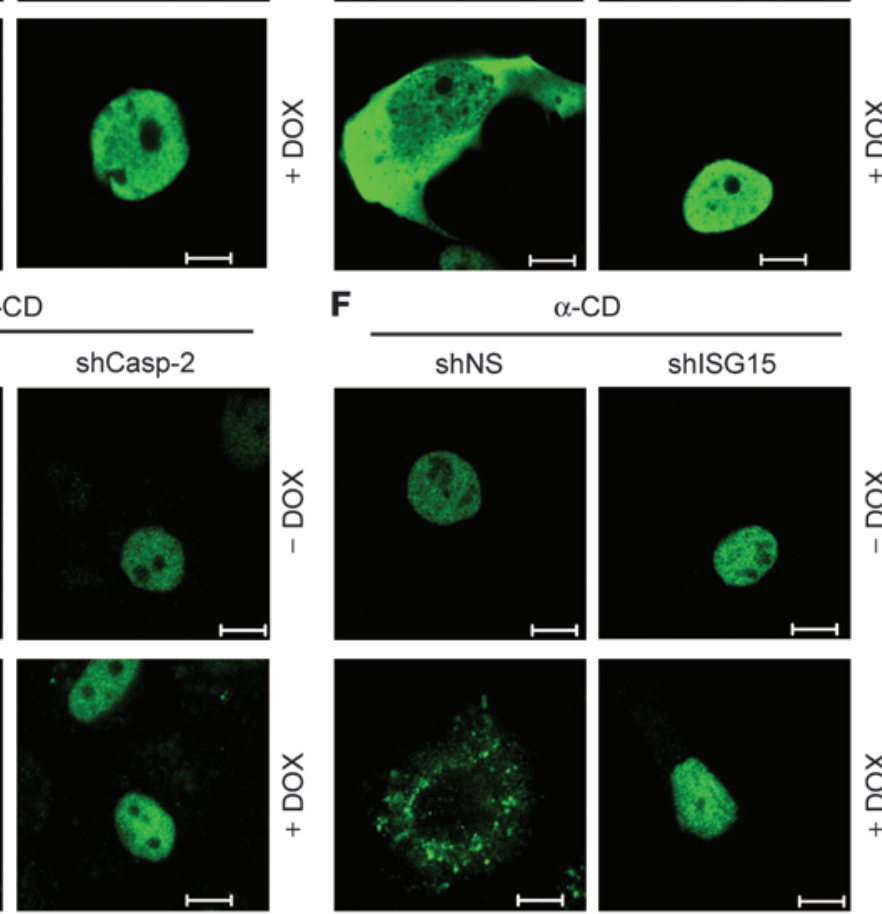

$\mathbf{F}$

shNS
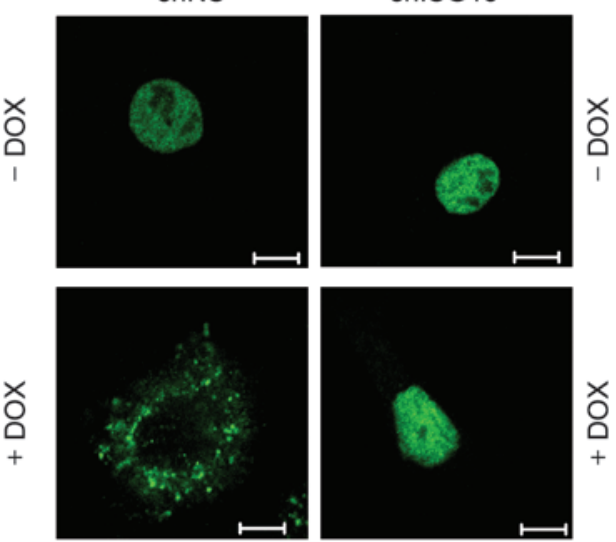

I $++++\ldots+-$ shNS

- - - + + + + + shISG15

$\begin{array}{llllllllll}0 & 6 & 12 & 24 & 0 & 6 & 12 & 24 & \mathrm{DOX}(\mathrm{h})\end{array}$

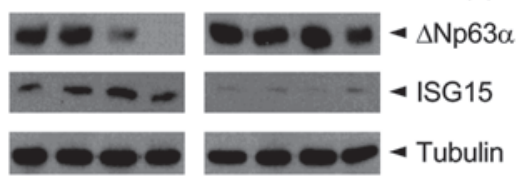

Figure 5

Requirement of ISGylation and caspase-2-mediated cleavage of $\Delta \mathrm{Np} 63 \alpha$ for cytoplasmic export of TI domain. (A and B) H1299 cells complemented with $\triangle N p 63 \alpha$ or KR were incubated with doxorubicin for 12 hours followed by staining with (A) anti-ND or (B) anti-CD antibody. (C) Cells complemented with HisMax-tagged $\triangle N p 63 \alpha$ or 3DA were incubated with doxorubicin followed by staining with anti-CD antibody. (D) HNSCC013 cells were incubated with doxorubicin in the absence or presence of Z-VDVAD-fmk followed by staining with anti-CD antibody. (E) HNSCC013 cells transfected with shNS or shCasp-2 were incubated with doxorubicin followed by staining with anti-CD antibody. (F) HNSCC013 cells transfected with shNS or shISG15 were incubated with doxorubicin followed by staining with anti-CD antibody. Scale bars: $10 \mu \mathrm{m}(\mathbf{A}-\mathbf{F})$. (G) Cells cultured as in D were subjected to IB with anti-ND or anti-CD antibody. (H) Cells cultured as in $\mathbf{E}$ were subjected to IB with anti- $\triangle$ Np63 $\alpha$ or anticaspase-2 antibody. (I) Cells cultured as in F were subjected to IB with anti- $\Delta$ Np63 $\alpha$ or anti-ISG15 antibody.

the cytoplasm, and this export was prevented by ISG15 depletion (Supplemental Figure 7B and see below). Collectively, these results indicate that doxorubicin-induced ISGylation and caspase-2-dependent cleavage of $\Delta \mathrm{Np} 63 \alpha$ in the nucleus lead to export of its TI domain to the cytoplasm.

ISGylation of TAp $63 \alpha$ promotes its transactivity. TAp $63 \alpha$ has $\mathrm{TI}$ domain in its $\mathrm{C}$ terminus in addition to the $\mathrm{N}$-terminal TA domain $(27,28)$. To address the possibility that doxorubicin induces ISGylation of TAp $63 \alpha$, which influences its transactivity, we incubated H1299 cells complemented with TAp63 $\alpha$ or its KR form with and without the drug. Like $\Delta \mathrm{Np} 63 \alpha$, TAp $63 \alpha$, but not KR, was ISGylated in response to doxorubicin (Figure 6A). Doxorubicin also induced cleavage of TAp $63 \alpha$, but not KR, and this proteolysis was abrogated by Z-VDVAD-fmk (Figure 6B). The cleaved TI domain of TAp63 $\alpha$ was then released to the cytoplasm (Figure 6C and Supplemental Figure 8). Consistently, doxorubi- 
A
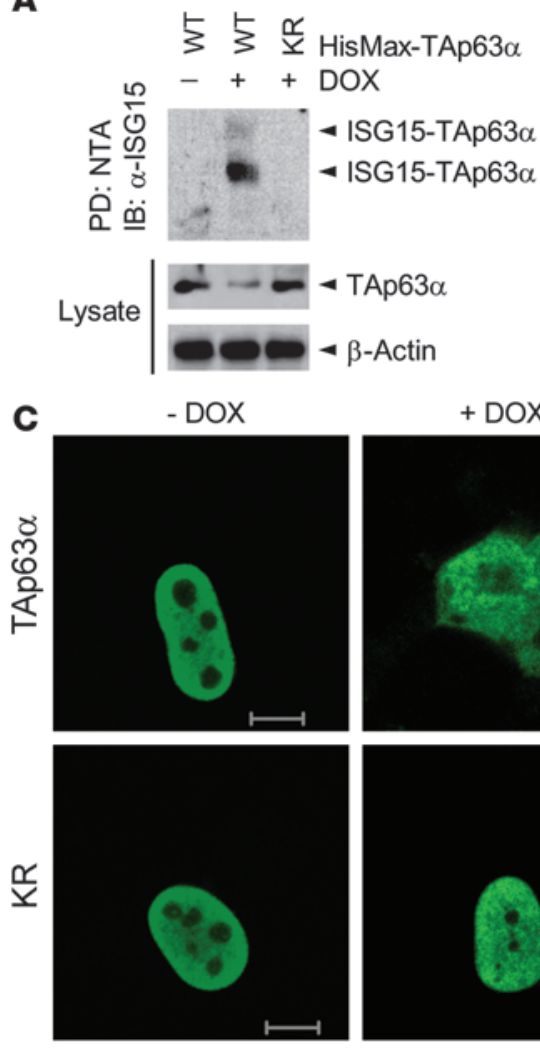

B

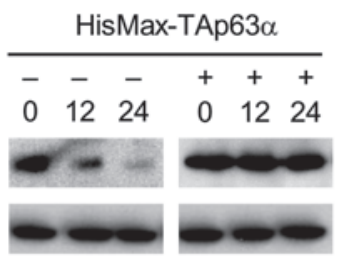

HisMax-KR

\begin{tabular}{llllllll}
\hline- & - & - & + & + & + & Z-VDVAD-fmk \\
0 & 12 & 24 & 0 & 12 & 24 & $\operatorname{DOX}(\mathrm{h})$
\end{tabular}

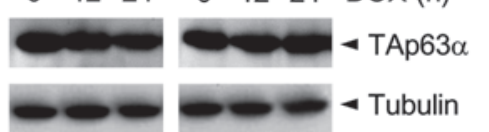

D

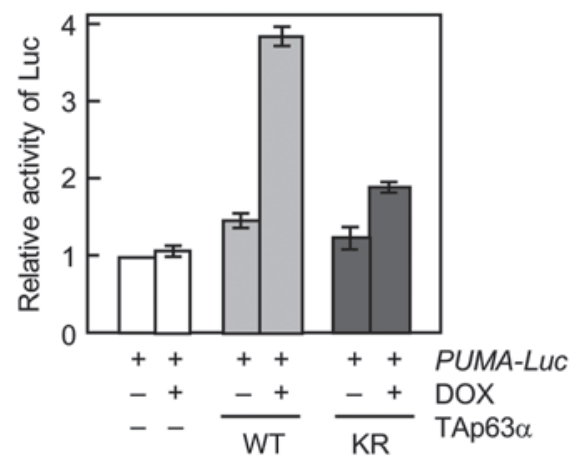

\section{Figure 6}

ISGylation of TAp63 $\alpha$ promotes its transactivity. (A) H1299 cells complemented with HisMax-tagged TAp63 $\alpha$ (WT) or KR were incubated for 12 hours with doxorubicin. Cell lysates were subjected to pulldown with NTA resins followed by IB with anti-ISG15 antibody. (B) Cells complemented with HisMax-tagged TAp63 $\alpha$ or KR were incubated with doxorubicin in the absence or presence of Z-VDVAD-fmk. Cell lysates were subjected to IB with anti-Xpress antibody. (C) Cells incubated as in A were stained with anti-CD antibody. Scale bars: $10 \mu \mathrm{m}$. (D) PUMA-Luc was transfected to cells with vectors expressing HisMax-tagged TAp63 $\alpha$ or KR. After incubation for 12 hours with or without doxorubicin, they were subjected to assay for luciferase. Enzyme activity is expressed relative to that seen with reporter vector alone in the absence of doxorubicin (assigned as 1.0). Data are mean \pm SD of 3 independent experiments.

cin markedly increased the transactivity of TAp63 $\alpha$, but much less so that of KR, as analyzed using PUMA-Luc (Figure 6D). These results indicate that doxorubicin-induced ISGylation, caspase-2-mediated cleavage, and export of TI domain to the cytoplasm relieve the suppressive function of TI domain within TAp $63 \alpha$, leading to promotion of TAp $63 \alpha$ transactivity.

IS Gylation of $\triangle N p 63 \alpha$ abrogates its dominant-negative function toward TAp63 $\gamma$. TAp $63 \gamma$ lacks TI domain and therefore acts as a potent transcriptional activator on p53-responsive target genes $(29,35)$. To determine the effect of $\Delta \mathrm{Np} 63 \alpha$ ISGylation on dominantnegative action toward TA isotypes, TAp63 $\gamma$ was expressed with $\triangle \mathrm{Np} 63 \alpha, \mathrm{KR}$, or 3DA in H1299 cells transfected with PUMA-Luc or PG13-Luc. In the absence of doxorubicin, all forms of $\Delta \mathrm{Np} 63 \alpha$ strongly inhibited the transactivity of TAp63 $\gamma$ (Figure 7, A and B). In its presence, both KR and 3DA persistently blocked TAp63 $\gamma$ transactivity, whereas $\Delta \mathrm{Np} 63 \alpha$ no longer inhibited it. These results indicate that doxorubicin-induced ISGylation and caspase-2-mediated cleavage of $\Delta \mathrm{Np} 63 \alpha$ abrogates its dominantnegative function toward TAp63\%.

ISGylation of $\triangle N p 63 \alpha$ abrogates its antiapoptotic function. To determine the effect of $\Delta \mathrm{Np} 63 \alpha$ ISGylation on its mitogenic and antiapoptotic functions, Hep3B cells that stably express $\Delta \mathrm{Np} 63 \alpha, \mathrm{KR}$, and 3DA were generated. In the absence of doxorubicin, all forms of $\Delta \mathrm{Np} 63 \alpha$ promoted cell proliferation (Supplemental Figure 9A); in its presence, both KR and 3DA persistently promoted cell growth, whereas $\Delta \mathrm{Np} 63 \alpha$ could no longer do so. Furthermore, TUNEL analysis revealed that doxorubicin abolished the antiapoptotic activity of $\Delta \mathrm{Np} 63 \alpha$, but not that of KR and 3DA (Supplemental Figure 9B). These results indicate that doxorubicininduced ISGylation and caspase-2-mediated cleavage of $\Delta \mathrm{Np} 63 \alpha$ abrogates its cell growth-promoting and antiapoptotic functions.

To confirm the effect of $\Delta \mathrm{Np} 63 \alpha$ ISGylation on its antiapoptotic function in cancer cells, ISG $15, \Delta \mathrm{Np} 63 \alpha$, or both in HNSCC013 cells were depleted by expressing shISG15 and shRNA specific to $\Delta \mathrm{Np} 63 \alpha(\operatorname{sh} \Delta \mathrm{Np} 63 \alpha)$. In control cells (i.e., transfected with shNS), doxorubicin-induced $\Delta \mathrm{Np} 63 \alpha$ cleavage occurred concurrently with induction of apoptotic events, such as caspase-3 activation and expression of Noxa, a proapoptotic protein (Figure 8A). Similar results were observed with $\Delta \mathrm{Np} 63 \alpha$ depletion. On the other hand, ISG15 knockdown causing $\Delta \mathrm{Np} 63 \alpha$ stabilization led to ablation of the apoptotic events. Knockdown of both ISG15 and $\Delta \mathrm{Np} 63 \alpha$, however, led to induction of the apoptotic events in response to doxorubicin. Consistently, ISG15 depletion strongly inhibited the doxo- 
A

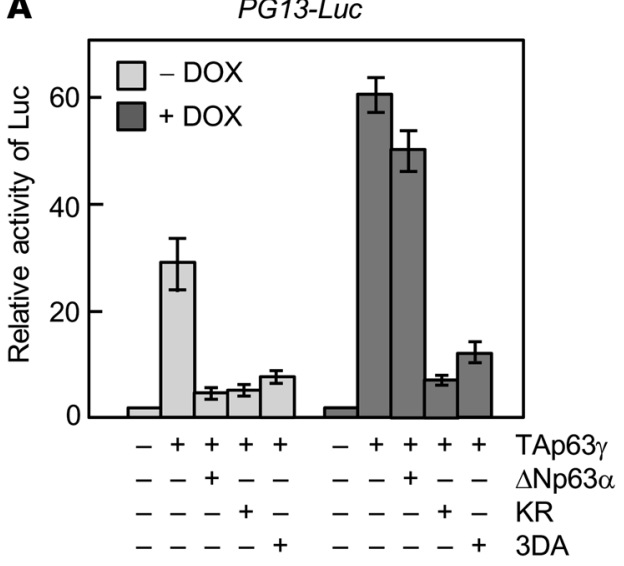

B

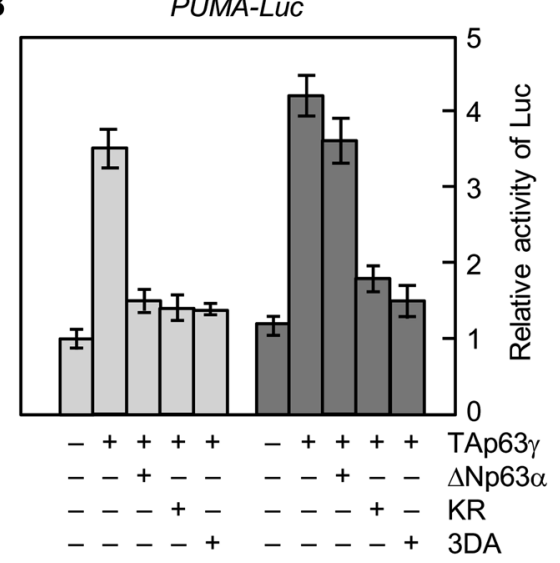

\section{Figure 7}

ISGylation of $\Delta \mathrm{Np} 63 \alpha$ abrogates its dominant-negative function toward TAp63 $\gamma . \Delta \mathrm{Np} 63 \alpha, \mathrm{KR}$, or 3DA was expressed in cells that had been complemented with TAp63 $\gamma$ and transfected with (A) PG13-LuC or (B) PUMA-Luc. After incubation for 12 hours with or without doxorubicin, cells were subjected to assay for luciferase. Enzyme activity is expressed relative to that seen with reporter vector alone in the absence of doxorubicin (assigned as 1.0). Data are mean \pm SD of 3 experiments. rubicin-mediated increase in the number of TUNEL-positive cells, and this inhibition was abolished by knockdown of both ISG15 and $\Delta \mathrm{Np} 63 \alpha$ (Figure $8 \mathrm{~B}$ ). To determine whether doxorubicin-induced $\Delta \mathrm{Np} 63 \alpha$ ISGylation indeed affects p63-mediated expression of proapoptotic proteins, ChIP assays were performed. Doxorubicin treatment led to TAp63 $\alpha$ recruitment to the Noxa promoter, which was prevented by ISG15 knockdown (Figure 8C). Conversely, drug treatment caused a marked decrease in the level of $\Delta \mathrm{Np} 63 \alpha$ bound to the promoter, which was attenuated by ISG15 knockdown. These results indicate that doxorubicin-induced ISGylation and subsequent cleavage of $\Delta \mathrm{Np} 63 \alpha$ is responsible for ablation of its antiapoptotic function in cancer cells.

To further assess the contribution of doxorubicin-induced $\Delta \mathrm{Np} 63 \alpha$ ISGylation to apoptosis of cancer cells, we searched for cells that are resistant to doxorubicin-mediated apoptosis. Of the available human epithelial tumor-derived cell lines with elevated $\Delta \mathrm{Np} 63 \alpha$ expression, we found that T47D cells derived from human breast cancer, unlike HCC1937 cells, were resistant to doxorubicin, as analyzed by cell growth and TUNEL assays (Figure 8, D and E). Because doxorubicin was unable to induce ISG15 expression, neither ISGylation nor destabilization of $\Delta \mathrm{Np} 63 \alpha$ was detected in T47D cells (Figure $8 \mathrm{~F}$ ). However, the drug was capable of inducing caspase-2 activation (Figure $8 \mathrm{G}$ ), which indicates that $\Delta \mathrm{Np} 63 \alpha$ ISGylation is required for its cleavage by activated caspase-2. These results suggest that doxorubicin resistance of T47D cells is due to their inability to induce ISG15 expression, but not to multidrug resistance that might have been acquired. However, further studies are required to clarify why T47D cells could not induce ISG15 induction in response to doxorubicin.

ISGylation of $\Delta N p 63 \alpha$ promotes oncogenic Ras-mediated senescence. In addition to the ability of TAp63 isotypes to promote expression of p53 downstream genes, they can function as tumor suppressors independently of p53 by inducing senescence (26). Moreover, it was recently demonstrated that downregulation of $\Delta \mathrm{Np} 63 \alpha$ is required for oncogenic Ras-induced senescence and that overexpression of $\Delta \mathrm{Np} 63 \alpha$ bypasses senescence and promotes stem-like proliferation and carcinoma development (45). To investigate the possible involvement of $\Delta \mathrm{Np} 63 \alpha$ ISGylation in oncogene-induced senescence, we transfected primary human keratinocytes with H-Ras-V12. Intriguingly, the Ras expression led to an increase in the level of ISGylated $\Delta \mathrm{Np} 63 \alpha$ as well as of
ISG15, concurrently with a marked decrease in $\Delta \mathrm{Np} 63 \alpha$ level (Supplemental Figure 10A). In addition, ISG15 knockdown prevented oncogenic Ras-mediated destabilization of $\Delta \mathrm{Np} 63 \alpha$ (Supplemental Figure 10B), which indicates that $\Delta \mathrm{Np} 63 \alpha$ ISGylation is required for its destabilization in response to Ras expression. Furthermore, ISG15 knockdown was sufficient to block oncogenic Ras-induced senescence, as determined by assaying senescence-associated $\beta$-galactosidase activity (Supplemental Figure 10, C and D). These results suggest that ISGylation and subsequent destabilization of $\Delta \mathrm{Np} 63 \alpha$, which would in turn activate TAp63 isotypes, is responsible for oncogenic Ras-mediated cellular senescence.

ISGylation of $\Delta N p 63 \alpha$ abrogates its oncogenic function. To determine the effect of $\Delta \mathrm{Np} 63 \alpha$ ISGylation on its oncogenic function, colony formation assays were performed using Hep3B cells that stably express $\Delta \mathrm{Np} 63 \alpha, \mathrm{KR}$, or 3DA. In the absence of doxorubicin, all $\Delta \mathrm{Np} 63 \alpha$ forms markedly increased colony number as well as size (Figure 9, A and B). In its presence, however, the ability of $\Delta \mathrm{Np} 63 \alpha$ - but not of KR or 3DA - to induce colony formation was drastically attenuated, implicating the role of $\Delta \mathrm{Np} 63 \alpha$ ISGylation in ablation of its oncogenic function. To determine whether $\Delta \mathrm{Np} 63 \alpha$ ISGylation indeed contributes to tumor suppression in vivo, we performed xenograft analysis. Without doxorubicin treatment, BALB/c nude mice injected with A549 cells expressing $\Delta \mathrm{Np} 63 \alpha, \mathrm{KR}$, or 3DA developed large tumors (Figure 9, $C$ and D, and Supplemental Figure 11A). In A549- $\Delta$ Np $63 \alpha$ xenografts, the drug treatment inhibited tumor growth, thereby reducing tumor sizes. In contrast, A549-KR or A549-3DA xenografts did not respond to doxorubicin, which indicates that ISGylation and caspase-2-mediated cleavage of $\Delta \mathrm{Np} 63 \alpha$ is required for doxorubicin-mediated tumor suppression.

To confirm the role of $\Delta \mathrm{Np} 63 \alpha$ ISGylation in tumor suppression, we generated ME180 cells (a human cervical cancer cell line) that stably express shISG15. Doxorubicin treatment led to ISGylation and destabilization of $\Delta \mathrm{Np} 63 \alpha$ in cells expressing shNS, but not in cells expressing shISG15 (Figure 9E). In the absence of doxorubicin, BALB/c nude mice injected with cells expressing either shNS or shISG15 developed large tumors (Figure 9F and Supplemental Figure 11B). On the other hand, doxorubicin treatment markedly decreased tumor growth in mice injected with cells expressing shNS, but not in mice injected with cells expressing shISG15, which again indicates that $\Delta \mathrm{Np} 63 \alpha$ ISGylation plays a key role in doxorubicin-mediated tumor suppression. 


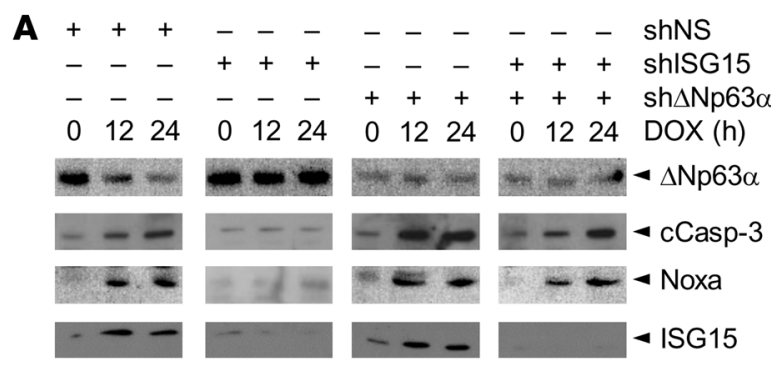

C
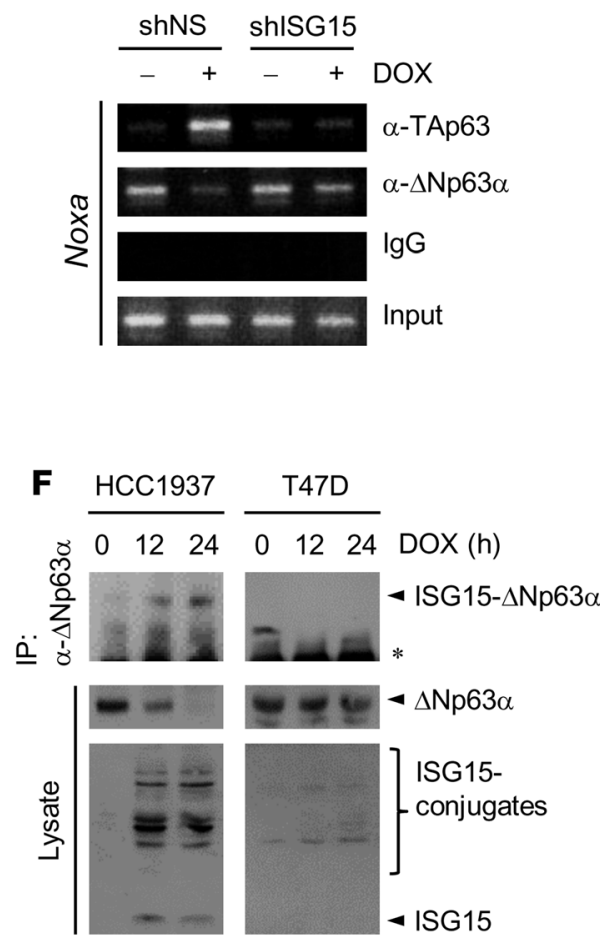

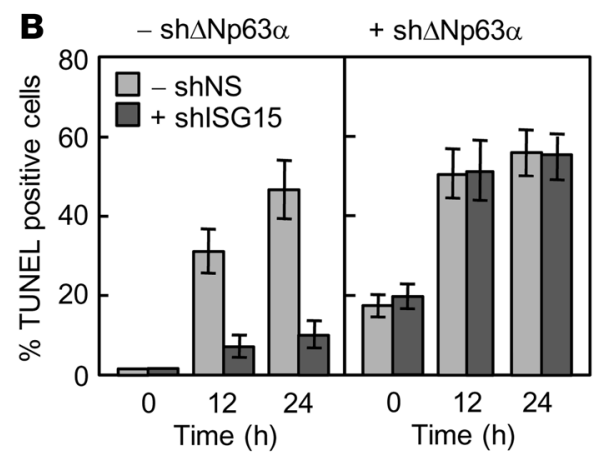

D

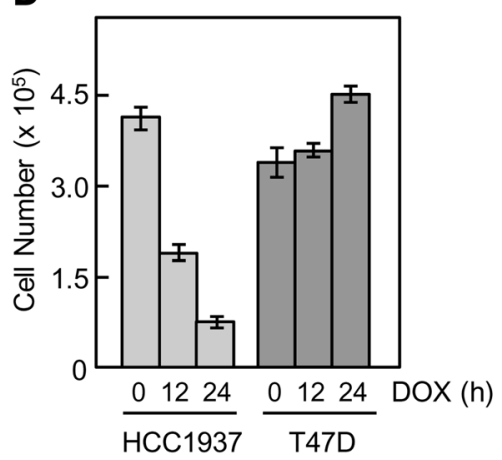

E

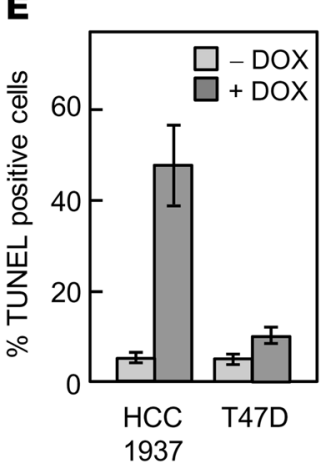

G $\quad 0 \quad 12 \quad 24 \quad \operatorname{DOX}(\mathrm{h})$

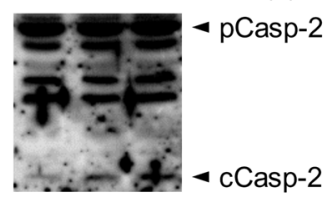

Figure 8

ISGylation of $\Delta$ Np63 $\alpha$ ablates its antiapoptotic function. (A and B) HNSCC013 cells transfected with shNS, shISG15, sh $\Delta$ Np63 $\alpha$, or both shISG15 and $\operatorname{sh} \triangle \mathrm{Np63} \alpha$ were incubated with doxorubicin, and cell lysates were subjected to (A) IB analysis and (B) TUNEL assay. (C) ME180 cells expressing shNS or shISG15 were incubated without or with doxorubicin for 24 hours, then subjected to ChIP using anti-TAp63 or anti- $\Delta$ Np63 $\alpha$ antibody. (D-G) HCC1937 and T47D cells were incubated with doxorubicin for 0, 12, or 24 hours. (D) Cell number. (E) TUNEL assay after 24 hours of culture. (F) IP with anti- $\Delta$ Np63 $\alpha$ antibody, followed by IB with anti-ISG15 antibody. Asterisk denotes IgG heavy chain. (G) IB with anti-caspase-2 antibody in T47D cells. Data are mean \pm SD of 3 experiments.

Finally, we examined whether doxorubicin-induced ISGylation and cleavage of $\Delta \mathrm{Np} 63 \alpha$ alters its subcellular localization in tumor tissues. Paraffin sections prepared from the tumors obtained as above were subjected to immunohistochemistry. When the sections from tumors derived by injecting cells expressing shNS were incubated with anti-ND antibody, intense nuclear staining of $\triangle \mathrm{Np} 63 \alpha$ was observed, regardless of doxorubicin treatment (Figure 9G). When incubated with anti-CD antibody, however, nuclear staining of $\Delta \mathrm{Np} 63 \alpha$ was markedly diminished, and cytoplasmic staining increased upon treatment with doxorubicin, but not without it. On the other hand, exclusive nuclear staining was evident in the section from tumors derived by injecting cells expressing shISG15, regardless of doxorubicin treatment. Moreover, subcellular fractionation of tumors derived by injecting cells expressing shNS showed that doxorubicin treatment caused the appearance of the C-terminal fragment of $\Delta \mathrm{Np} 63 \alpha$ in the cytoplasmic fractions (Figure 9H). On the other hand, little or no C-terminal fragment of $\Delta \mathrm{Np} 63 \alpha$ was seen in the cytoplasmic fractions obtained from tumors derived by injecting cells expressing shISG15, regardless of doxorubicin treatment. Collectively, our findings indicate that doxorubicin-induced ISGylation and caspase-2-mediated cleavage of $\Delta \mathrm{Np} 63 \alpha-$ and subsequent release of TI domain to the cytoplasm - ablates its oncogenic function.

\section{Discussion}

Based on our present findings, we propose a model for the mechanism by which ISGylation exerts its anticancer action toward tumors with high levels of $\Delta \mathrm{Np} 63 \alpha$ in response to doxorubicin 
A Control
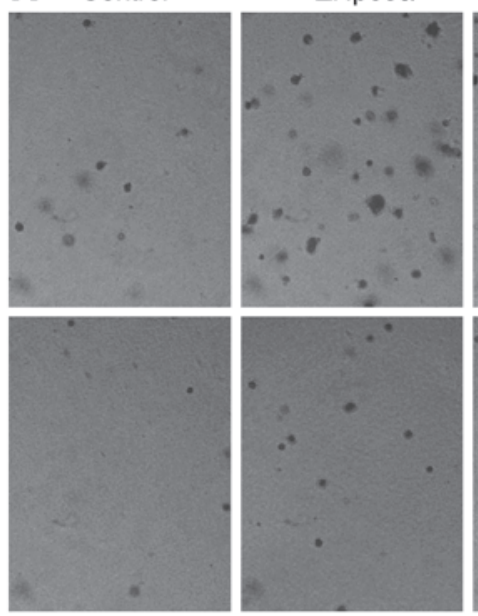

$\mathrm{KR}$
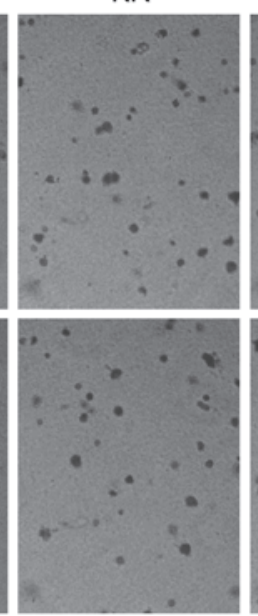

3DA

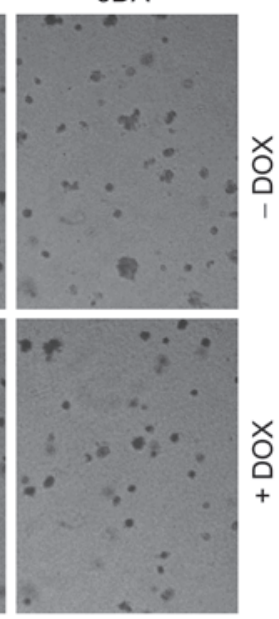

B

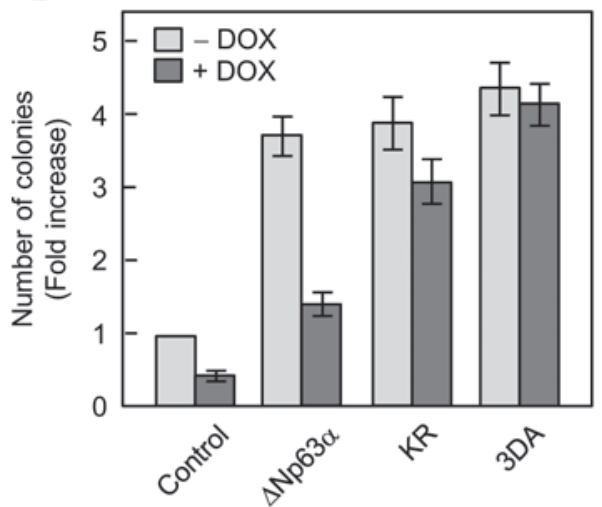

C

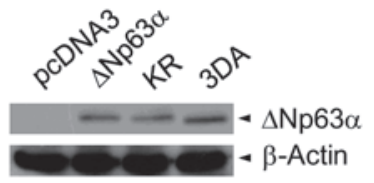

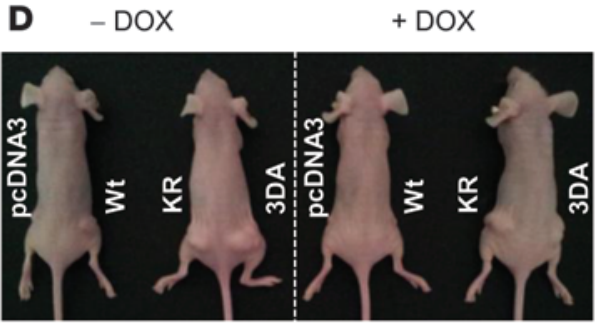

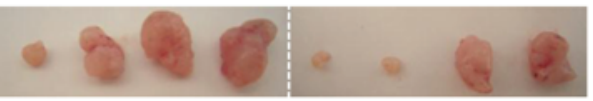

E

IP: $\alpha-\Delta N p 63 \alpha$

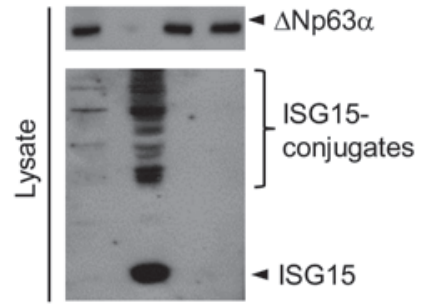

shISG15

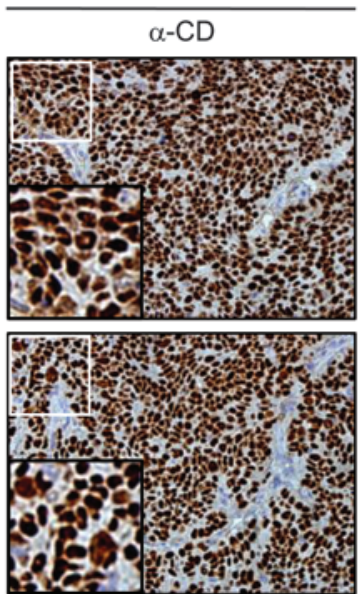

shNS

G $\alpha$-ND
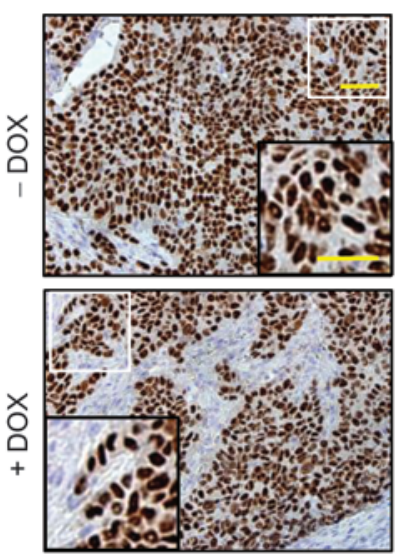
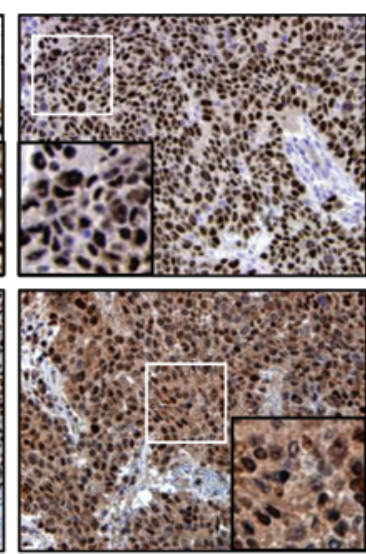

$\mathbf{F}$

$\frac{\text { shNS }}{-\mathrm{DOX}+\mathrm{DOX}} \frac{\text { shISG15 }}{-\mathrm{DOX}+\mathrm{DOX}}$

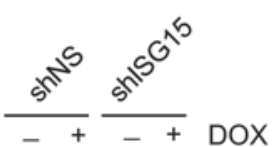

- ISG15- $\Delta \mathrm{Np} 63 \alpha$
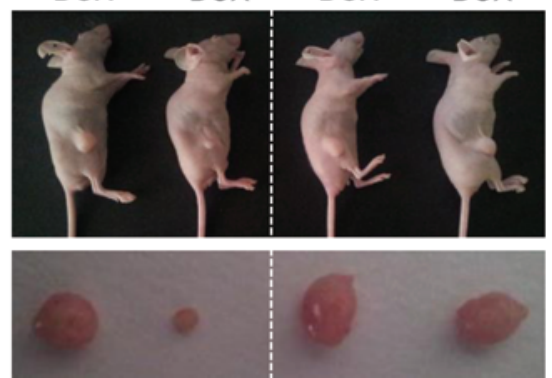

H

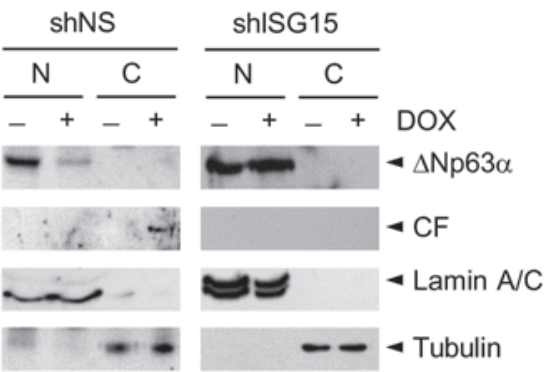

\section{Figure 9}

ISGylation of $\Delta \mathrm{Np} 63 \alpha$ ablates its oncogenic function. (A and B) Hep3B cells stably expressing $\Delta N p 63 \alpha$, KR, or 3DA were grown in soft agar. 5 weeks later, colonies were stained with crystal violet (A), and the number of colonies per plate was determined (B). Results are expressed relative to the number in cells transfected with empty vector (control) in the absence of doxorubicin (assigned as 1.0). Data are mean \pm SD of 3 experiments. (C) A549 cells stably expressing $\Delta N p 63 \alpha, K R$, or 3DA were subjected to IB with anti- $\Delta N p 63 \alpha$ antibody. pcDNA3 indicates an empty vector. (D) Cells $\left(5 \times 10^{6}\right)$ prepared as in C were subcutaneously injected to BALB/c nude mice. Doxorubicin treatment was as described in Methods. 35 days after injection, mice were sacrificed, and tumors were dissected out. (E) ME180 cells stably expressing shNS or shISG15 were incubated with doxorubicin. Cell lysates were subjected to IP with anti- $\Delta$ Np63 $\alpha$ antibody followed by IB with anti-ISG15 antibody. (F) Cells $\left(6.5 \times 10^{6}\right)$ prepared as in $\mathbf{E}$ were injected to mice. Doxorubicin treatment was as described in Methods. (G) Paraffin sections of tumors derived from mice as in $\mathbf{F}$ were subjected to immunohistochemical staining with anti-ND or anti-CD antibody. Insets show magnified views of the boxed regions. Scale bars: $100 \mu \mathrm{m}$. (H) Tumors from $\mathbf{F}$ were fractionated into the nuclear $(\mathrm{N})$ and cytoplasmic $(\mathrm{C})$ fractions followed by IB with anti-ND or anti-CD antibody. 
(Supplemental Figure 12). In cancer cells with elevated expression of $\Delta \mathrm{Np} 63 \alpha$, the transactivities of TA isotypes, which induce cell cycle arrest and apoptosis, would be suppressed by dominantnegative action of $\Delta \mathrm{Np} 63 \alpha$. This suppression makes cells resistant to apoptosis, causing uncontrolled cell proliferation and tumor formation. Upon treatment with doxorubicin, $\Delta \mathrm{Np} 63 \alpha$ would be ISGylated and cleaved by caspase-2. The N-terminal fragment of $\Delta \mathrm{Np} 63 \alpha$, which no longer has TI domain, would be unable to suppress the transactivation by TA isotypes. The C-terminal fragment of $\Delta \mathrm{Np} 63 \alpha$, although retaining TI domain, could not show its suppressive function, since it is exported to the cytoplasm. Under the same conditions, TAp $63 \alpha$ would also be ISGylated and cleaved by caspase-2, followed by release of its C-terminal TI domain to the cytoplasm. Thus, the N-terminal fragment of TAp $63 \alpha$ having TA domain, but no longer containing TI domain, would be relieved from self-suppression and thereby capable of inducing expression of its downstream apoptotic genes. The transactivities of other TA isotypes that constitutively lack TI domain (e.g., TAp63 $\gamma$ ) would also be promoted, as the nucleus is devoid of TI domain. These findings indicate that ISGylation and caspase-2-mediated cleavage of $\Delta \mathrm{Np} 63 \alpha$ in response to doxorubicin serve as a critical mechanism in the ablation of the antiapoptotic and oncogenic functions of $\Delta \mathrm{Np} 63 \alpha$ as well as in the promotion of the transactivities of TA isotypes.

In contrast to doxorubicin and camptothecin, cisplatin - also a well-known anticancer drug - did not induce ISGylation or cleavage of $\Delta \mathrm{Np} 63 \alpha$. However, it has been shown that cisplatin induces c-Abl-mediated phosphorylation of TAp73, which leads to dissociation of the $\Delta \mathrm{Np} 63 \alpha / \mathrm{TAp} 73$ protein complex and promotion of TAp73-dependent transactivity and apoptosis (37). Thus, cisplatin, like doxorubicin, impairs the dominant-negative function of $\Delta \mathrm{Np} 63 \alpha$ toward TA isotypes, although it shows no effect on $\Delta \mathrm{Np} 63 \alpha$ stability, unlike doxorubicin.

ISG15 has been suggested to be a tumor suppressor, based on the findings that chemotherapeutics such as camptothecin increase ISG15 levels $(17-19,55)$. In addition, shRNA-mediated knockdown of either ISG15 or UbcH8 in breast cancer cells decreases their sensitivity to camptothecin (66). UBE1L has also been suggested to act as a tumor suppressor. Retinoic acid induces UBE1L and subsequent ISGylation of the PML/RAR $\alpha$ fusion protein $(16,67,68)$, whose accumulation is a characteristic of acute promyelocytic leukemia. ISGylation of PML/RAR $\alpha$ results in its destabilization by proteasome-mediated degradation, thus overcoming oncogenic potential of the fusion protein, although it is unclear whether ISGylation acts in the same way that ubiquitin signals to proteasome. UBE1L also promotes cyclin D1 ISGylation, and this modification leads to the destabilization of cyclin D1, which indicates that UBE1L confers growth suppression by targeting cyclin D1 (19) and suggests that the tumor-suppressive role of UBE1L could also operate in tumor cells that do not necessarily express high levels of $\Delta \mathrm{Np} 63 \alpha$. However, it is unknown how the stability of cyclin D1 is affected by its ISGylation. In addition, UBE1L and $p 53$ double-deficient mice are prone to develop thymic lymphomas, compared with p53 singledeficient mice (69). Here we demonstrated that ISGylation plays a critical role in conferring chemosensitivity to epithelial tumors with elevated expression of $\Delta \mathrm{Np} 63 \alpha$. Doxorubicin, in addition to camptothecin, induced increased expression of ISG15, UBE1L, and $\mathrm{UbcH} 8$, and this induction led to ISGylation and caspase-2mediated cleavage of $\Delta \mathrm{Np} 63 \alpha$, thus abrogating its ability to pro- mote anchorage-independent cell growth and tumor growth. Our findings provide the first unequivocal evidence to our knowledge for involvement of ISGylation of a specific target protein, $\Delta \mathrm{Np} 63 \alpha$, in tumor suppression.

An important unanswered question is how doxorubicin upregulates expression of ISG15, UBE1L, and UbcH8. It is known that ISG15 is a target gene of p53 (70). However, it is unlikely that doxorubicin-mediated upregulation of ISG15, UBE1L, and UbcH8 is under direct control of p53, because doxorubicin leads to an increase in the transcript levels of ISG15-conjugating machinery in HNSCC013 and HCC1937 cells, both of which are known to express mutated nonfunctional p53 $(37,71)$. An alternative possibility is the involvement of Notch signaling, given that this pathway plays an important role in tumor suppression in cells like keratinocytes (72). $\Delta \mathrm{Np} 63 \alpha$ is highly expressed in proliferating keratinocytes (33), and its expression is suppressed with differentiation (72-75). On the other hand, Notch signaling promotes keratinocyte differentiation, in part through the activation of IKK $\alpha$ and NF-кB-mediated transcription (76-78). Moreover, Notch inhibits $\Delta \mathrm{Np} 63 \alpha$ expression in differentiating keratinocytes (75). In addition, p63 is negatively regulated by NF- $\kappa \mathrm{B}(75)$, which is induced in differentiating keratinocytes $(79,80)$, suggesting that suppression of $\mathrm{p} 63$ by Notch involves NF- $\mathrm{\kappa B}$ activation. Importantly, ISG15 is a target gene of NF-кB (6). Therefore, it seems possible that Notch-mediated NF- $\mathrm{KB}$ activation promotes the induction of ISG15-conjugating machinery in doxorubicin-treated epithelial cancer cells as well as in differentiating keratinocytes, thereby leading to downregulation of $\Delta \mathrm{Np} 63 \alpha$.

Caspase- 2 is known to be cleaved and activated by a protein complex called PIDDosome, which contains caspase-2, PIDD, and adaptor protein RAIDD (59). Caspase-2 has also been shown to form a large nuclear protein complex called DNA-PKcs-PIDDosome, which contains caspase-2, PIDD, and DNA-PKcs, but not RAIDD (81). Moreover, DNA damage induced by $\gamma$-irradiation triggers the phosphorylation of caspase- 2 within its prodomain by the kinase, leading to caspase- 2 activation. However, specific target-mediated cellular functions of caspase-2 remained unclear, since only a few substrates of the protease - including Golgin-160, $\alpha$ II-spectrin, PKC $\delta$, and Bid - have been identified so far $(62,82-85)$. In the present study, we demonstrated that doxorubicin induced caspase- 2 activation and that $\Delta \mathrm{Np} 63 \alpha$ was a specific substrate of activated caspase-2. This proteolytic event led to cytoplasmic export of TI domain of $\Delta \mathrm{Np} 63 \alpha$ and in turn abrogated the dominant-negative function of the oncogenic protein. Importantly, loss of caspase- 2 has been shown to increase the ability of cells to acquire a transformed phenotype and become malignant (86), implicating the role of caspase- 2 as a tumor suppressor. Recently, it was shown that DNA damage triggers caspase- 2 activation and that the activated caspase- 2 cleaves $\mathrm{Mdm} 2$, resulting in p53 stabilization (87). Thus, it seems likely that the tumor-suppressive function of caspase- 2 is mediated by its ability to cleave $\Delta \mathrm{Np} 63 \alpha$ as well as $\mathrm{Mdm} 2$.

It remains unknown how caspase- 2 is activated in response to doxorubicin. Neither is the mechanical basis for preferential interaction of ISGylated $\Delta \mathrm{Np} 63 \alpha$ with activated caspase- 2 over its unmodified form yet clear. It may be possible that, like $\gamma$-irradiation (28), doxorubicin treatment triggers the phosphorylation of caspase-2 by DNA-PKc for its activation. With respect to the preferential interaction of ISGylated $\Delta \mathrm{Np} 63 \alpha$, the conjugated ISG15 may provide a better structural environment for interaction of $\Delta \mathrm{Np} 63 \alpha$ with activated caspase- 2 or recruit unknown proteins 
that could help the recognition of activated caspase-2. Further studies are required to resolve these important questions.

\section{Methods}

Materials. Polyclonal anti-ISG15 antibody was raised in rabbits by injecting purified ISG15 protein. Antibodies against TAp $63 \alpha, \Delta \mathrm{Np} 63 \alpha$ (Santa Cruz), caspase-2 (R\&D Systems), caspase-9, caspase-3 (Cell Signaling), and Noxa (Abcam) were used. Monoclonal anti-Myc antibody was produced from 9E10 hybridoma. Doxorubicin (Sigma-Aldrich), camptothecin, cisplatin (Calbiochem), Z-VDVAD-fmk (Biovision), Z-DEVD-fmk, and Z-VAD-fmk (R\&D Systems) were used. cDNAs of $\Delta \mathrm{Np} 63 \alpha$, TAp63 $\alpha$, and TAp63 $\gamma$ were provided by H.S. Lee (Seoul National University). cDNAs for $\Delta \mathrm{Np} 63 \alpha$ and its deletion mutants were cloned into pcDNA4-HisMax. Human ISG15 cDNAs were amplified from total RNA of IFN- $\beta$-treated A549 cells and subcloned into pFlagCMV10.

shRNA sequences. shCasp-2, shISG15, and $\operatorname{sh} \Delta \mathrm{Np} 63 \alpha$ as well as shRNAs specific to their empty vectors were purchased from Openbiosystems; shCasp-3 was purchased from Sigma-Aldrich. Sequences were as follows: shCasp-2, CCGGCTAGTCACCCTCTTCAAGCTTCTCGAGAAGCTTGAAGAGGGTGACTAGTTTTT; shISG15, CCGGGCAGACCGTGGCCCACCTGAACTCGAGTTCAGGTGGGCCACGGTCTGCTTTTT; $\operatorname{sh} \Delta \mathrm{Np} 63 \alpha$, CTCCCCAGATGATGAACTGTTATAGTGAAGCCACAGATGTATAACAGTTCATCTGGGGAT; shCasp-9, AATGCAGATTTGGCTTACA; shCasp-3, CCGGGCGAATCAATGGACTCTGGAACTCGAGTTCCAGAGTCCATTGATTCGCTTTTT.

Cell culture and transfection. UBE1L MEFs were provided by D.E. Zhang (UCSD, San Diego, California, USA), and HNSCC013 cells were provided by D. Sidransky and E. Ratovotski (Johns Hopkins University, Baltimore, Maryland, USA). HNSCC013, T47D, HCC1937, and ME180 cells were cultured in RPMI 1640 containing 10\% FBS. FaDu cells (ATCC) were maintained in MEM containing 10\% FBS. HeLa, H1299, A549, and Hep3B cells were cultured in DMEM (JBI) supplemented with 10\% FBS. MCF10A cells were maintained in DMEM/F12 (Invitrogen) supplemented with 5\% horse serum, $20 \mathrm{ng} / \mathrm{ml} \mathrm{EGF,} 10 \mu \mathrm{g} / \mathrm{ml}$ insulin, $1 \mathrm{ng} / \mathrm{ml}$ cholera toxin, and $100 \mu \mathrm{g} / \mathrm{ml}$ hydrocortisone. Plasmids and shRNAs were transfected to cells using Lipofectamine with PLUS reagent (Invitrogen). Protein knockdown efficiency ranged $80 \%-100 \%$, as verified by IB analysis. Primary human keratinocytes were cultured in growth medium with added human keratinocyte growth supplements (Lonza). Cells were kept constantly subconfluent to avoid triggering of differentiation. Cells were transfected using Cytofect transfection reagents (Cell applications).

IP and nitrilotriacetic acid (NTA) pulldown analysis. Cells were lysed in $50 \mathrm{mM}$ Tris- $\mathrm{HCl}$ ( $\mathrm{pH} 8$ ) containing $150 \mathrm{mM} \mathrm{NaCl}, 1 \%$ Triton X-100 or $0.5 \%$ NP-40, $1 \mathrm{mM} \mathrm{PMSF}$, and $1 \times$ protease inhibitor cocktail (Roche). Cell lysates were incubated with appropriate antibodies for 2 hours at $4{ }^{\circ} \mathrm{C}$ and then with $50 \mu \mathrm{l}$ of $50 \%$ slurry of protein A-Sepharose for 1 hour. For pulldown analysis, cell lysates were prepared as above and treated with $\mathrm{Ni}^{2+}$ NTA agarose (Qiagen).

Immunocytochemistry. Cells were grown on coverslips. 2 days after transfection, they were fixed by incubation for 10 minutes with $3.7 \%$ paraformaldehyde in PBS. Cells were washed 3 times with PBS containing $0.1 \%$ Triton X-100, permeabilized with $0.5 \%$ Triton X-100 in PBS for 5 minutes, and treated with $3 \%$ BSA in PBS for 1 hour. They were then incubated for 1 hour with appropriate antibodies. After washing with PBS containing $0.1 \%$ Triton X-100, cells were incubated for 1 hour with FITC-conjugated secondary antibody in PBS containing 3\% BSA. Cells were observed using a confocal laser scanning microscope (Carl Zeiss-LSM510). Images were processed using Photoshop (Adobe).

Immunohistochemistry. Dissected xenograft tumors were fixed in $4 \%$ paraformaldehyde overnight at $4{ }^{\circ} \mathrm{C}$, paraffin embedded, and sectioned
(4- $\mu \mathrm{m}$ thickness). Paraffin sections were incubated for 1 hour at $37^{\circ} \mathrm{C}$ with anti-ND (4A4; Santa Cruz) or anti-CD (H129; Santa Cruz) antibodies. Sections were incubated with horseradish peroxidase-conjugated goat anti-mouse or goat anti-rabbit secondary antibody. Signals were detected using 3,3-diaminobenzidine as a substrate. Slides were then counterstained with hematoxylin.

Senescence-associated $\beta$-galactosidase assay. Cells were grown in 6-well culture plates, washed with PBS, fixed in $2 \%$ paraformaldehyde and $0.25 \%$ glutaraldehyde for 10 minutes, and stained for 24 hours at $37^{\circ} \mathrm{C}$ with $\beta$-galactosidase staining solution $(40 \mathrm{mM}$ citric acid/sodium phosphate, $\mathrm{pH}$ 6.0; $150 \mathrm{mM} \mathrm{NaCl} ; 2 \mathrm{mM} \mathrm{MgCl}_{2} ; 5 \mathrm{nM}$ potassium ferrocyanide; $5 \mathrm{nM}$ potassium ferricyanide; and $1 \mathrm{mg} / \mathrm{ml} 5$-bromo-4-chloro-3-indolyl- $\beta$-Dgalactopyranoside; Cell Signaling). The reaction was stopped by replacing the staining solution with $70 \%$ glycerol.

ChIP. ME180 cells that had been treated with doxorubicin $(0.1 \mu \mathrm{M})$ for 24 hours were subjected to ChIP analysis (88). Briefly, TAp63-DNA and $\triangle \mathrm{Np} 63$-DNA complexes were subjected to IP using antibodies against TAp63 (D-20; Santa Cruz) and $\Delta \mathrm{Np} 63$ (N-16; Santa Cruz), respectively. DNAs were extracted from the precipitates, and aliquots of the extracts were subjected to PCR. Primers used for Noxa were as follows: 5' primer, ACGATGTTCTTTCTGGCTGG; 3' primer, GCTTTGACCATCTGCAAACG.

Luciferase assay. H1299 cells seeded in 12-well plates were transfected with appropriate expression vectors and reporter plasmids. After treatment with doxorubicin for 12 hours, lysates were analyzed using Luciferase Assay System (Promega) as recommended by the manufacturer.

Subcellular fractionation. Cells suspended in buffer A (10 mM Tris- $\mathrm{HCl}$, pH 7.9; 1 mM DTT; 350 mM sucrose; PMSF; and inhibitor cocktail) were incubated for 20 minutes on ice with swirling. They were lysed using a dounce homogenizer and centrifuged at $3,000 \mathrm{~g}$ for 5 minutes. The soluble fraction was again centrifuged at $15,000 \mathrm{~g}$ for 30 minutes, and the supernatant was used as the cytoplasmic fraction. The pellets were resuspended in 5 volumes of buffer A containing $0.3 \% \mathrm{NP}-40$ and mixed with equal volume of buffer B (10 mM Tris-HCl, pH 8.0; 2.2 M sucrose; 5 mM magnesium acetate; and $0.1 \mathrm{mM}$ EDTA). Purified nuclei were collected by centrifugation at $15,000 \mathrm{~g}$ for 20 minutes and resuspended in buffer $\mathrm{C}$ (20 mM HEPES, pH 7.9; 1 mM EDTA; 1 mM EGTA; 1 mM DTT; 400 mM NaCl; 1 mM PMSF; and inhibitor cocktail). They were then lysed by vigorous vortexing and dilution with buffer $C$. In the case of subcellular fractionation of dissected xenograft tumors, tumors were treated with papain $(20 \mu \mathrm{g} / \mathrm{ml})$ and DNase $(10 \mathrm{U} / \mu \mathrm{l})$ for 40 minutes at $37^{\circ} \mathrm{C}$ and then dissociated mechanically by titration with a glass Pasteur pipette.

Cell growth, colony formation, and tumorigenesis assay. Hep3B cells stably expressing HisMax-tagged $\Delta \mathrm{Np} 63 \alpha, \mathrm{KR}$, or $3 \mathrm{DA}$ were selected by incubation with zeocin $(50 \mu \mathrm{g} / \mathrm{ml})$. Individual clones were picked and verified by Western blot analysis (data not shown). For cell growth assay, they were seeded in 12 wells in triplicate, harvested, and stained with trypan blue. For colony formation assay, cells $\left(1 \times 10^{4}\right)$ were resuspended in $0.35 \%$ agar in DMEM supplemented with $10 \% \mathrm{FBS}$ and then overlaid on $0.7 \%$ agar in the same medium in 6-well plates. The plates were incubated at $37^{\circ} \mathrm{C}$ in $5 \% \mathrm{CO}_{2}$ for 5 weeks. Colonies were stained with $0.005 \%$ crystal violet and counted. BALB/c nude mice were purchased from Orient Bio Inc. For in vivo tumorigenesis assay, A549 cells $\left(5 \times 10^{6}\right)$ stably expressing $\Delta \mathrm{Np} 63 \alpha$, KR, or 3DA or ME180 cells $\left(6.5 \times 10^{6}\right)$ stably expressing shNS or shISG15 were subcutaneously injected into the upper thigh of one or both legs of 6-week-old BALB/c nude mice. On the third day after injection, mice began receiving twice weekly i.p. injections of PBS or doxorubicin $(1.25 \mathrm{mg} / \mathrm{kg})$ for 3 weeks. They were monitored regularly for tumor growth. Tumor volumes were calculated as $(a \times a \times b) / 2$, in which $a$ is the smallest diameter and $b$ the largest. At the end of the experiments, mice were killed, and tumors were dissected. 
Statistics. All values are given as mean \pm SD. Statistical comparisons were made by 2 -tailed Student's $t$ test. A $P$ value less than 0.05 was considered statistically significant.

Study approval. All animal procedures were approved by the IACUC of Seoul National University.

\section{Acknowledgments}

We thank D.E. Zhang, D. Sidransky, and E. Ratovotski for providing cells and H.S. Lee for cDNAs. This work was supported by grants from the National Research Foundation of Korea (NRF-
2005-084-C00025 and M10533010001-05N3301). H.M. Yoo and J.M. Park were the recipients of the BK21 fellowship.

Received for publication November 2, 2011, and accepted in revised form May 9, 2012.

Address correspondence to: Chin Ha Chung, School of Biological Sciences, Seoul National University, 56-1 Shillim-dong, Kwanak-gu, Seoul 151-742, Republic of Korea. Phone: 82.2.880.6693; Fax: 82.2.871.9193; E-mail: chchung@snu.ac.kr.
1. Haas AL, Ahrens P, Bright PM, Ankel H. Interferon induces a 15-kilodalton protein exhibiting marked homology to ubiquitin. J Biol Chem. 1987; 262(23):11315-11323.

2. Kim MJ, Latham AG, Krug RM. Human influenza viruses activate an interferon-independent transcription of cellular antiviral genes: outcome with influenza A virus is unique. Proc Natl Acad Sci US A. 2002;99(15):10096-10101.

3. Farrell PJ, Broeze RJ, Lengyel P. Accumulation of an mRNA and protein in interferon-treated Ehrlich ascites tumor cells. Nature. 1979;279(5713):523-525.

4. Loeb KR, Haas AL. The interferon-inducible 15-kDa ubiquitin homolog conjugates to intracellular proteins. J Biol Chem. 1992;267(11):7806-7813.

5. Yuan W, Krug RM. Influenza B virus NS1 protein inhibits conjugation of the interferon (IFN)induced ubiquitin-like ISG15 protein. EMBO J. 2001;20(3):362-371.

6. Li J, et al. Novel NEMO/IkappaB kinase and NFkappa $\mathrm{B}$ target genes at the pre-B to immature $\mathrm{B}$ cell transition. J Biol Chem. 2001;276(21):18579-18590.

7. Malakhova O, Malakhov M, Hetherington C, Zhang DE. Lipopolysaccharide activates the expression of ISG15-specific protease UBP43 via interferon regulatory factor 3. J Biol Chem. 2002; 277(17):14703-14711.

8. Kim KI, Giannakopoulos NV, Virgin HW, Zhang DE. Interferon-inducible ubiquitin E2, Ubc8, is a conjugating enzyme for protein ISGylation. $\mathrm{Mol}$ Cell Biol. 2004;24(21):9592-9600.

9. Zhao C, et al. The UbcH8 ubiquitin E2 enzyme is also the E2 enzyme for ISG15, an IFN-alpha/betainduced ubiquitin-like protein. Proc Natl Acad Sci US A. 2004;101(20):7578-7582.

10. Wong JJ, Pung YF, Sze NS, Chin KC. HERC5 is an IFN-induced HECT-type E3 protein ligase that mediates type I IFN-induced ISGylation of protein targets. Proc Natl Acad Sci U S A. 2006; 103(28):10735-10740.

11. Zou W, Zhang DE. The interferon-inducible ubiquitin-protein isopeptide ligase (E3) EFP also functions as an ISG15 E3 ligase. J Biol Chem. 2006; 281(7):3989-3994

12. Malakhov MP, Kim KI, Malakhova OA, Jacobs BS, Borden EC, Zhang DE. High-throughput immunoblotting. Ubiquitin-like protein ISG15 modifies key regulators of signal transduction. J Biol Chem. 2003;278(19):16608-16613.

13. Zhao C, Denison C, Huibregtse JM, Gygi S, Krug RM. Human ISG15 conjugation targets both IFNinduced and constitutively expressed proteins functioning in diverse cellular pathways. Proc Natl Acad Sci U S A. 2005;102(29):10200-10205.

14. Jeon YJ, et al. ISG15 modification of filamin B negatively regulates the type I interferon-induced JNK signaling pathway. EMBO Rep. 2009;10(4):374-380.

15. Jeon YJ, Yoo HM, Chung CH. ISG15 and immune diseases. Biochim Biophys Acta. 2010;1802(5):485-496.

16. Pitha-Rowe I, Hassel BA, Dmitrovsky E. Involvement of UBE1L in ISG15 conjugation during retinoid-induced differentiation of acute promyelocytic leukemia. J Biol Chem. 2004;279(18):18178-18187.

17. McLaughlin PM, et al. The ubiquitin-activating enzyme E1-like protein in lung cancer cell lines. Int J Cancer. 2000;85(6):871-876.

18. Kok K, et al. A gene in the chromosomal region $3 \mathrm{p} 21$ with greatly reduced expression in lung cancer is similar to the gene for ubiquitin-activating enzyme. Proc Natl Acad Sci U S A. 1993;90(13):6071-6075.

19. Feng $Q$, et al. UBE1L causes lung cancer growth suppression by targeting cyclin D1. Mol Cancer Ther. 2008;7(12):3780-3788.

20. Levine AJ, Tomasini R, McKeon FD, Mak TW, Melino $G$. The p53 family: guardians of maternal reproduction. Nat Rev Mol Cell Biol. 2011;12(4):259-265.

21. Melino G. p63 is a suppressor of tumorigenesis and metastasis interacting with mutant p53. Cell Death Differ. 2011;18(9):1487-1499.

22. Flores ER, et al. p63 and p73 are required for p53-dependent apoptosis in response to DNA damage. Nature. 2002;416(6880):560-564.

23. Suh EK, et al. p63 protects the female germ line during meiotic arrest. Nature. 2006;444(7119):624-628.

24. Flores ER, et al. Tumor predisposition in mice mutant for p63 and p73: evidence for broader tumor suppressor functions for the p53 family. Cancer Cell. 2005;7(4):363-373.

25. Keyes WM, et al. p63 heterozygous mutant mice are not prone to spontaneous or chemically induced tumors. Proc Natl Acad Sci U S A. 2006; 103(22):8435-8440

26. Guo X, et al. TAp63 induces senescence and suppresses tumorigenesis in vivo. Nat Cell Biol. 2009; 11(12):1451-1457.

27. Serber Z, et al. A C-terminal inhibitory domain controls the activity of $\mathrm{p} 63$ by an intramolecular mechanism. Mol Cell Biol. 2002;22(24):8601-8611.

28. Deutsch GB, et al. DNA damage in oocytes induces a switch of the quality control factor TAp63alpha from dimer to tetramer. Cell. 2011;144(4):566-576.

29. Yang A, et al. p63, a p53 homolog at 3q27-29, encodes multiple products with transactivating, death-inducing, and dominant-negative activities. Mol Cell. 1998;2(3):305-316.

30. Sayan BS, et al. Cleavage of the transactivationinhibitory domain of p63 by caspases enhances apoptosis. Proc Natl Acad Sci U S A. 2007; 104(26):10871-10876.

31. Carroll DK, et al. p63 regulates an adhesion programme and cell survival in epithelial cells. Nat Cell Biol. 2006;8(6):551-561.

32. Mills AA, Zheng B, Wang XJ, Vogel H, Roop DR, Bradley A. p63 is a p53 homologue required for limb and epidermal morphogenesis. Nature. 1999;398(6729):708-713.

33. Yang A, et al. p63 is essential for regenerative proliferation in limb, craniofacial and epithelial development. Nature. 1999;398(6729):714-718.

34. Osada $\mathrm{M}$, et al. Cloning and functional analysis of human p51, which structurally and functionally resembles p53. Nat Med. 1998;4(7):839-843.

35. Osada M, et al. Differential recognition of response elements determines target gene specificity for $\mathrm{p} 53$ and p63. Mol Cell Biol. 2005;25(14):6077-6089.

36 . Hibi $\mathrm{K}$, et al. AIS is an oncogene amplified in squamous cell carcinoma. Proc Natl Acad Sci U S A. 2000; 97(10):5462-5467.
37. Leong CO, Vidnovic N, DeYoung MP, Sgroi D, Ellisen LW. The p63/p73 network mediates chemosensitivity to cisplatin in a biologically defined subset of primary breast cancers. J Clin Invest. 2007; 117(5):1370-1380.

38. Massion PP, et al. Significance of $\mathrm{p} 63$ amplification and overexpression in lung cancer development and prognosis. Cancer Res. 2003;63(21):7113-7121.

39. Ghioni P, Bolognese F, Duijf PH, Van Bokhoven H, Mantovani R, Guerrini L. Complex transcriptional effects of $\mathrm{p} 63$ isoforms: identification of novel activation and repression domains. Mol Cell Biol. 2002;22(24):8659-8668.

40. Wu G, et al. DeltaNp63alpha and TAp63alpha regulate transcription of genes with distinct biological functions in cancer and development. Cancer Res. 2003;63(10):2351-2357.

41. Candi E, et al. Differential roles of $\mathrm{p} 63$ isoforms in epidermal development: selective genetic complementation in p63 null mice. Cell Death Differ. 2006; 13(6):1037-1047.

42. Yang A, et al. Relationships between p63 binding, DNA sequence, transcription activity, and biological function in human cells. Mol Cell. 2006; 24(4):593-602.

43. Vigano MA, et al. New p63 targets in keratinocytes identified by a genome-wide approach. EMBO J. 2006;25(21):5105-5116.

44. Koster MI, et al. p63 induces key target genes required for epidermal morphogenesis. Proc Natl Acad Sci U S A. 2007;104(9):3255-3260.

45. Keyes WM, et al. DeltaNp63alpha is an oncogene that targets chromatin remodeler Lsh to drive skin stem cell proliferation and tumorigenesis. Cell Stem Cell. 2011;8(2):164-176.

46. Liefer KM, Koster MI, Wang XJ, Yang A, McKeon F, Roop DR. Down-regulation of p63 is required for epidermal UV-B-induced apoptosis. Cancer Res. 2000;60(15):4016-4020.

47. Westfall MD, Joyner AS, Barbieri CE, Livingstone $\mathrm{M}$, Pietenpol JA. Ultraviolet radiation induces phosphorylation and ubiquitin-mediated degradation of DeltaNp63alpha. Cell Cycle. 2005;4(5):710-716.

48. Ghioni P, et al. The protein stability and transcriptional activity of p63alpha are regulated by SUMO-1 conjugation. Cell Cycle. 2005;4(1):183-190.

49. Fomenkov A, et al. RACK1 and stratifin target DeltaNp63alpha for a proteasome degradation in head and neck squamous cell carcinoma cells upon DNA damage. Cell Cycle. 2004;3(10):1285-1295.

50. Bakkers J, Camacho-Carvajal M, Nowak M, Kramer C, Danger B, Hammerschmidt M. Destabilization of DeltaNp63alpha by Nedd4-mediated ubiquitination and Ubc9-mediated sumoylation, and its implications on dorsoventral patterning of the zebrafish embryo. Cell Cycle. 2005;4(6):790-800.

51. Rossi M, et al. Itch/AIP4 associates with and promotes p63 protein degradation. Cell Cycle. 2006;5(16): 1816-1822.

52. Rossi $\mathrm{M}$, et al. The E3 ubiquitin ligase Itch controls the protein stability of p63. Proc Natl Acad Sci US A. 2006;103(34):12753-12758.

53. Li Y, Zhou Z, Chen C. WW domain-containing E3 ubiquitin protein ligase 1 targets p 63 transcription 
factor for ubiquitin-mediated proteasomal degradation and regulates apoptosis. Cell Death Differ. 2008;15(12):1941-1951.

54. Peschiaroli A, Scialpi F, Bernassola F, El Sherbiniel S, Melino G. The E3 ubiquitin ligase WWP1 regulates DeltaNp63-dependent transcription through Lys63 linkages. Biochem Biophys Res Commun. 2010;402(2):425-430.

55. Liu M, Hummer BT, Li X, Hassel BA. Camptothecin induces the ubiquitin-like protein, ISG15, and enhances ISG15 conjugation in response to interferon. J Interferon Cytokine Res. 2004;24(11):647-654.

56 . Ohwada $S$, et al. Interferon potentiates antiproliferative activity of CPT-11 against human colon cancer xenografts. Cancer Lett. 1996;110(1-2):149-154.

57. Ratovitski EA, Patturajan M, Hibi K, Trink B, Yamaguchi K, Sidransky D. p53 associates with and targets Delta Np63 into a protein degradation pathway. Proc Natl Acad Sci U S A. 2001;98(4):1817-1822.

58. Lassus P, Opitz-Araya X, Lazebnik Y. Requirement for caspase- 2 in stress-induced apoptosis before mitochondrial permeabilization. Science. 2002; 297(5585):1352-1354.

59. Tinel A, Tschopp J. The PIDDosome, a protein complex implicated in activation of caspase- 2 in response to genotoxic stress. Science. 2004; 304(5672):843-846.

60. Bergeron L, et al. Defects in regulation of apoptosis in caspase-2-deficient mice. Genes Dev. 1998; 12(9):1304-1314

61. Colussi PA, Harvey NL, Kumar S. Prodomain-dependent nuclear localization of the caspase-2 (Nedd2) precursor. A novel function for a caspase prodomain. J Biol Chem. 1998;273(38):24535-24542.

62. Mancini M, et al. Caspase-2 is localized at the Golgi complex and cleaves golgin-160 during apoptosis. J Cell Biol. 2000;149(3):603-612.

63. Zhivotovsky B, Samali A, Gahm A, Orrenius S Caspases: their intracellular localization and translocation during apoptosis. Cell Death Differ. 1999;6(7):644-651.

64. Butt AJ, Harvey NL, Parasivam G, Kumar S. Dimerization and autoprocessing of the Nedd2 (caspase-2) precursor requires both the prodomain and the carboxyl-terminal regions. J Biol Chem. 1998; 273(12):6763-6768.

65. Read SH, Baliga BC, Ekert PG, Vaux DL, Kumar S. A novel Apaf-1-independent putative caspase-2 activation complex. J Cell Biol. 2002;159(5):739-745

66. Desai SD, et al. ISG15 as a novel tumor biomarker for drug sensitivity. Mol Cancer Ther. 2008; 7(6):1430-1439.

67. Shah SJ, et al. UBE1L represses PML/RAR\{alpha by targeting the PML domain for ISG15ylation. Mol Cancer Ther. 2008;7(4):905-914.

68. Kitareewan S, et al. UBE1L is a retinoid target that triggers PML/RARalpha degradation and apoptosis in acute promyelocytic leukemia. Proc Natl Acad SciU S A. 2002;99(6):3806-3811.

69. Yin X, Cong X, Yan M, Zhang DE. Alteration of tumor spectrum by ISGylation in p53-deficient mice. Cancer Biol Ther. 2009;8(12):1167-1172.

70. Hummer BT, Li XL, Hassel BA. Role for p53 in gene induction by double-stranded RNA. J Virol. 2001; 75(16):7774-7777.

71. Patturajan $\mathrm{M}$, et al. DeltaNp63 induces betacatenin nuclear accumulation and signaling. Cancer Cell. 2002;1(4):369-379.

72. Dotto GP. Crosstalk of Notch with p53 and p63 in cancer growth control. Nat Rev Cancer. 2009; 9(8):587-595.

73. Yi R, Poy MN, Stoffel M, Fuchs E. A skin microRNA promotes differentiation by repressing 'stemness'. Nature. 2008;452(7184):225-229.

74. Lena AM, et al. miR-203 represses 'stemness' by repressing DeltaNp63. Cell Death Differ. 2008; 15(7):1187-1195

75. Nguyen BC, et al. Cross-regulation between Notch and p63 in keratinocyte commitment to differentiation. Genes Dev. 2006;20(8):1028-1042.

76. Lowell S, Jones P, Le Roux I, Dunne J, Watt FM. Stimulation of human epidermal differentiation by delta-notch signaling at the boundaries of stemcell clusters. Curr Biol. 2000;10(9):491-500.

77. Nickoloff BJ, Qin JZ, Chaturvedi V, Denning MF, Bonish B, Miele L. Jagged-1 mediated activation of notch signaling induces complete maturation of human keratinocytes through NF-kappaB and
PPARgamma. Cell Death Differ. 2002;9(8):842-855.

78. Rangarajan A, et al. Notch signaling is a direct determinant of keratinocyte growth arrest and entry into differentiation. EMBO J. 2001; 20(13):3427-3436.

79. Seitz CS, Lin Q, Deng H, Khavari PA. Alterations in NF-kappaB function in transgenic epithelial tissue demonstrate a growth inhibitory role for NF-kappaB. Proc Natl Acad Sci U S A. 1998;95(5):2307-2312

80. van Hogerlinden M, Rozell BL, Ahrlund-Richter L, Toftgard R. Squamous cell carcinomas and increased apoptosis in skin with inhibited Rel/ nuclear factor-kappaB signaling. Cancer Res. 1999; 59(14):3299-3303.

81. Shi M, et al. DNA-PKcs-PIDDosome: a nuclear caspase-2-activating complex with role in $\mathrm{G} 2 / \mathrm{M}$ checkpoint maintenance. Cell. 2009;136(3):508-520.

82. Gao Z, Shao Y, Jiang X. Essential roles of the Bcl-2 family of proteins in caspase-2-induced apoptosis. J Biol Chem. 2005;280(46):38271-38275.

83. Rotter B, Kroviarski Y, Nicolas G, Dhermy D, Lecomte MC. AlphaII-spectrin is an in vitro target for caspase-2, and its cleavage is regulated by calmodulin binding. Biochem J. 2004;378(pt 1):161-168.

84. Xue D, Shaham S, Horvitz HR. The Caenorhabditis elegans cell-death protein CED-3 is a cysteine protease with substrate specificities similar to those of the human CPP32 protease. Genes Dev. 1996; 10(9):1073-1083.

85. Panaretakis $\mathrm{T}$, et al. Doxorubicin requires the sequential activation of caspase-2, protein kinase Cdelta, and c-Jun NH2-terminal kinase to induce apoptosis. Mol Biol Cell. 2005;16(8):3821-3831.

86. Ho LH, Taylor R, Dorstyn L, Cakouros D, Bouillet P, Kumar S. A tumor suppressor function for caspase-2. Proc Natl Acad Sci U S A. 2009;106(13):5336-5341.

87. Oliver TG, et al. Caspase-2-mediated cleavage of $\mathrm{Mdm} 2$ creates a $\mathrm{p} 53$-induced positive feedback loop. Mol Cell. 2011;43(1):57-71.

88. Baek SH, Ohgi KA, Rose DW, Koo EH, Glass CK, Rosenfeld MG. Exchange of N-CoR corepressor and Tip60 coactivator complexes links gene expression by NF-kappaB and beta-amyloid precursor protein. Cell. 2002;110(1):55-67. 